\title{
Integrating genetic dependencies and genomic alterations across pathways and cancer types
}

\author{
Tae Yoon Park ${ }^{* 1,2}$, Mark D.M. Leiserson ${ }^{* 3}$, Gunnar W. Klau ${ }^{* 4}$, and Benjamin J. Raphael ${ }^{1,2}$ \\ ${ }^{1}$ Department of Computer Science, Princeton University \\ ${ }^{2}$ Lewis-Sigler Institute for Integrative Genomics, Princeton University \\ ${ }^{3}$ Department of Computer Science and Center for Bioinformatics and Computational Biology, University of \\ Maryland, College Park \\ ${ }^{4}$ Algorithmic Bioinformatics, Heinrich Heine University Düsseldorf, Germany
}

\begin{abstract}
Recent genome-wide CRISPR-Cas9 loss-of-function screens have identified genetic dependencies across many cancer cell lines. Associations between these dependencies and genomic alterations in the same cell lines reveal phenomena such as oncogene addiction and synthetic lethality. However, comprehensive characterization of such associations is complicated by complex interactions between genes across genetically heterogeneous cancer types. We introduce SuperDendrix, an algorithm to identify differential dependencies across cell lines and to find associations between differential dependencies and combinations of genetic alterations and cell-type-specific markers. Application of SuperDendrix to CRISPR-Cas9 loss-of-function screens from 554 cancer cell lines reveals a landscape of associations between differential dependencies and genomic alterations across multiple cancer pathways in different combinations of cancer types. We find that these associations respect the position and type of interactions within pathways with increased dependencies on downstream activators of pathways, such as NFE2L2 and decreased dependencies on upstream activators of pathways, such as CDK6. SuperDendrix also reveals dozens of dependencies on lineage-specific transcription factors, identifies cancer-type-specific correlations between dependencies, and enables annotation of individual mutated residues.
\end{abstract}

\section{Introduction}

A key problem in cancer biology is to identify the genes that cancer cells depend on for their growth and survival advantage. This knowledge both informs our understanding of cancer development and also suggests therapeutic targets [1, 2, 3]. While some of these cancer-essential genes are altered by somatic mutations and thus identified by high-throughput DNA sequencing [4, 5, 6], other genes are rarely or not mutated in cancer, such as lineage-specific transcription factors or master regulators [7, 8, 9, 10]. An alternative approach to identify cancer dependencies is to perturb genes in in vitro cancer models, such as cell lines, and measure growth or viability following such perturbations. Genes whose perturbation results in a change in viability reveal potential cancer-specific gene dependencies. Recent technologies such as genome-wide pooled RNAi [11] or CRISPR [12, 13] loss-of-function screens enable high-throughput genome-wide perturbation screens. Projects such as DRIVE [14] and the Cancer Dependency Map (DepMap) [15, 16] have applied these technologies to hundreds of cancer cell lines and identified genes that are essential in specific cancer cell lines, often referred to as conditionally essential genes, or differential dependencies. Combining differential dependencies with genomic mutations identified in the same cell lines has revealed several contextspecific dependencies including examples of oncogene addiction [17, 18] and synthetic lethality [19, 20].

${ }^{*}$ These authors contributed equally 
Recently, several studies have attempted to systematically identify associations between differential dependencies and genomic alterations using data from large-scale RNAi and CRISPR datasets [14, 16, 21, 22, 23, 24, 25, 26]. The computational approaches used in these studies can be grouped into two classes. The first class of approaches attempt to identify associations between differential dependencies and single genomic biomarkers [14, 16, 21, 22, 23, 24]. These approaches recapitulate many of the classic oncogene addiction and synthetic lethal interactions, as well as a few additional associations. However, the restriction to a single biomarker limits the statistical power to detect associations with rare genomic alterations occurring in a small subset of cell lines. It is well known that driver mutations in cancer exhibit a "long tail" with relatively few frequently mutated genes and many rarely mutated genes [4, 5] of driver mutations in cancer. Thus, it is reasonable to assume that rare genomic alterations may also be associated with genetic dependencies. One explanation for the long tail of driver mutations is that many of the functions perturbed in cancer involve interactions between many genes in pathways and networks [5, 27, 28]. A genetic dependency may similarly be associated with genomic alterations in many genes, and thus examining single alterations or single genes may miss many important associations.

The second class of computational approaches attempt to identify associations between differential dependencies and sets of multiple biomarkers [15, 25, 26]. For example, Tsherniak et al. [15] used a random forest classifier to predict dependencies in the DepMap dataset from genomic alterations. While thousands of associations are reported, many of the reported associations are with gene expression markers and other frequent events, which is not surprising since the classifier skews toward explaining the most frequent associations. REVEALER [25] leverages the observation that driver mutations in the same pathway tend to be mutually exclusive across tumors, i.e., that few tumors have more than one driver mutation in a given pathway [29, 30, 31]. This method generalizes earlier approaches that identify sets of mutually exclusive mutations in cancer genomes [31, 32, 33, 34, 35], by focusing the search for sets of mutually exclusive mutations to cell lines with the the highest dependency scores. However, REVEALER's iterative search does not scale to the analysis of thousands of gene perturbations and genomic alterations [26]. Furthermore, REVEALER does not evaluate statistical significance of the reported associations, leading to many false positive associations. Recently, UNCOVER addressed the scalability issues of REVEALER using a combinatorial optimization approach [26]. Unfortunately, when applied to large-scale RNAi or CRISPR datasets, UNCOVER predicts hundreds-to-thousands of associations, and the quality of such predictions is generally unknown. These issues are due to three factors. Spurious associations are a significant challenge in analyzing large-scale RNAi or CRISPR screens, since the number of phenotypes (gene perturbations) and number of features (genomic alterations) are orders of magnitude larger than the number of samples. This challenge is further exacerbated when searching for sets of multiple biomarkers as the number of such sets is massive and the optimal set is unknown a priori.

We introduce a new algorithm, SuperDendrix, to identify sets of approximately mutually exclusive genomic alteration and cell type features that are associated with differential dependencies from large-scale perturbation experiments. SuperDendrix includes several key features: (1) a principled approach to identify and score differential dependencies using a 2-component mixture model; (2) a combinatorial model and optimization algorithm to find feature sets associated with differential dependencies; (3) a model selection criterion to select the size of the associated set and a robust statistical test that accounts for different frequencies of genomic alterations across samples.

We apply SuperDendrix to identify associations between genotypes and differential dependencies in a large-scale CRISPR-Cas9 screen dataset from DepMap of 17,634 gene knockouts across 558 cancer cell lines from 31 cancer types. We identify 32 associations between sets of alterations and differential dependencies, and an additional 117 associations that include cancer-type features. Many of these associations group into well-known cancer pathways including the NFE2L2, RB1, MAPK, and Wnt pathways. We observe that associations between differential dependencies and genomic alterations within a pathway respect the topology and regulatory logic of the interactions within the pathway. Specifically, we find that cell lines 
containing oncogenic mutations in a gene upstream in a pathway (either activating mutations in an oncogene or inactivating mutations in a tumor suppressor genes) often have increased dependencies on genes downstream in same pathway. These associations generalize the phenomenon of oncogene addiction to oncogene pathway addiction [17, 18]. On the other hand, we find that oncogenic mutations in genes that are downstream in a pathway are often associated with decreased dependency on genes upstream in the same pathway. SuperDendrix also identifies associations between differential dependencies and cancer types, most prominently dependencies involving lineage-specific transcription factors and a previously unreported association between TCF3 dependency in multiple myleoma or B-cell lymphomas with mutations in ID3 or $M E F 2 B$.

SuperDendrix is a rigorous computational approach to identify associations between gene dependencies, genomic alterations, and cell-type features in large-scale RNAi and CRISPR screens. SuperDendrix's high specificity focuses experimental efforts on a small number of the most promising associations. SuperDendrix software is publicly available at https://github.com/raphael-group/superdendrix and results on DepMap datasets are available through a web portal https://superdendrix-explorer.lrgr.io/.

\section{Results}

\section{SuperDendrix}

We introduce SuperDendrix, an algorithm to identify sets of binary features such as genomic alterations and/or cell types that are (approximately) mutually exclusive and associated with a quantitative phenotype. SuperDendrix is generally applicable to any quantitative phenotype, but in this work we focus on the phenotype of cell viability change following genome-wide CRISPR-Cas9 loss-of-function screens. The inputs to SuperDendrix are the following.

- Cell viability measurements from a genome-wide CRISPR-Cas9 loss-of-function screens. We represent these measurements in a phenotype matrix $P$ where each entry $p_{g j}$ of $P$ the indicates viability of cell line $j$ when gene $g$ is knocked out. Each of these scores quantify the dependency of a cell line on a gene. We refer to the dependency scores for a gene across cell lines (i.e. row of $P$ ) as a dependency profile.

- A list of somatic alterations in each cell line. Here, we analyze somatic missense and nonsense mutations.

- (Optionally) Categorical information (e.g. cell type) of each cell line.

SuperDendrix consists of three modules (Fig. 19): (1) Scoring differential dependencies and selecting genomic and cell-type features. (2) Finding feature sets associated with differential dependencies. (3) Evaluating the statistical significance of associations. We briefly describe these three modules below. Further details are in Methods

(1) Scoring differential dependencies and selecting genomic and cell-type features. The first module in SuperDendrix has two steps: (i) scoring differential dependencies from the dependency scores; (ii) selecting the genomic alteration and cell-type features that will be evaluated for association. In the first step, we derive a differential dependency profile for each gene knockout (row of $P$ ). This profile quantifies the magnitude of the effect on the gene knockout on each cell line relative to a background distribution. We assume that the dependency scores $p_{g 1}, \ldots, p_{g n}$ for knockout $g$ are generated from two populations: a background population that is unaffected by the knockout, and a responsive population that is affected by the knockout. We fit 
a

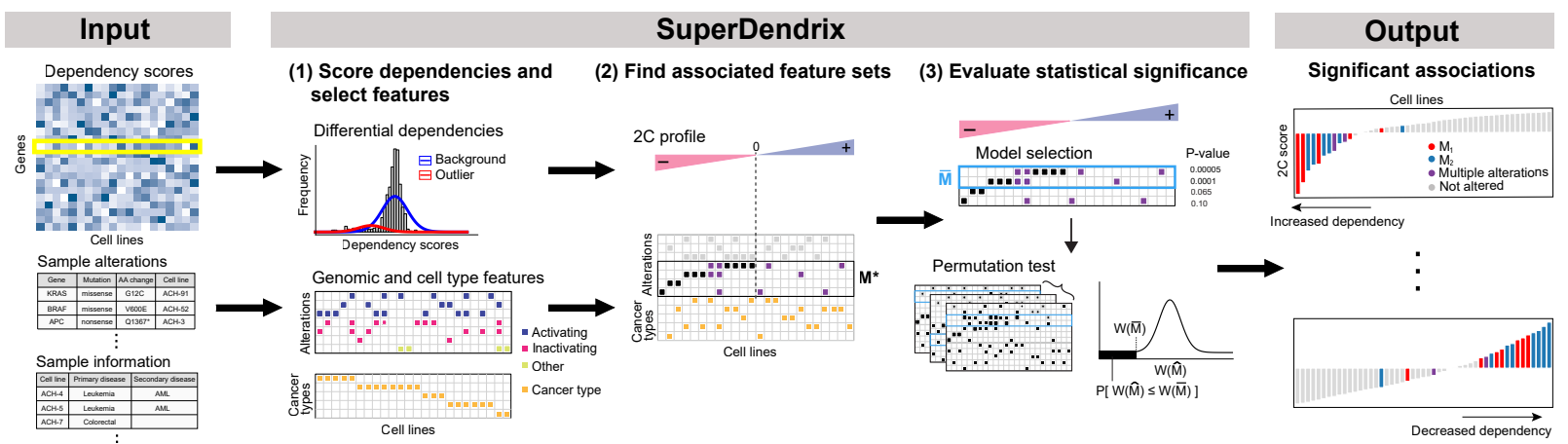

b
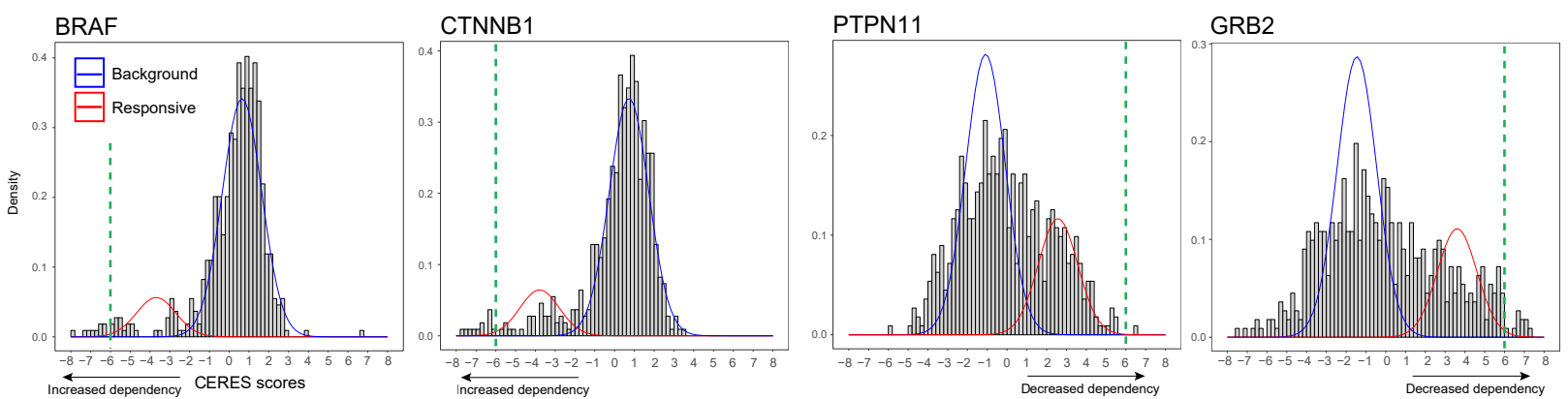

Figure 1: Overview of SuperDendrix. (a) SuperDendrix inputs are dependency scores of gene knockouts from CRISPR-Cas9 screens, genomic alterations, and optionally, cell-type features. In the first step, SuperDendrix scores differential dependencies - genes whose dependency scores are better fit by a mixture distribution of two components - and also constructs a genome alteration and cell-type feature matrix. In the second step, SuperDendrix finds a subset $M^{*}$ of features that maximize the SuperDendrix weight $W(M)$. In the third step, SuperDendrix performs model selection to define a subset $\bar{M}$ of features that substantially contribute to weight, and computes statistical significance of weight $W(\bar{M})$ using a permutation test. Associations with false discovery rate $(\mathrm{FDR}) \leq 0.2$ are output, and include associations between features and increased dependency on profile (top right) and between features and decreased dependency on features (bottom right). (b) Examples of $2 \mathrm{C}$ differential dependencies from DepMap data that result from fitting the dependency scores with a mixture model. Blue curve is the background component, and red curve is the responsive component. Green dashed lines indicate $6 \sigma$ criterion of Tsherniak et al. [15], which identifies only a subset of cell lines that are responsive to knockout. BRAF and CTNNB1 are examples of profiles with increased dependency in response to knockout while PTPN11 and GRB2 are examples of decreased dependency. 
a two-component mixture model to the dependency scores $p_{g 1}, \ldots, p_{g n}$, and decide whether the score distribution is better fit by one-component or two-components using the Bayesian Information Criterion (BIC). In the case where the two-component fit is preferred, we say that the cell lines are differentially dependent with respect to the gene knockout $g$, or that gene $g$ is a differential dependency. We define the differential dependency score, or $2 C$ score, $d_{g j}=\log \frac{\operatorname{Pr}\left(z_{g j}=2 \mid p_{g j}\right)}{\operatorname{Pr}\left(z_{g j}=1 \mid p_{g j}\right)}$ for cell line $j$ as the $\log$ ratio of the posterior probabilities that cell line $j$ is from component $2\left(z_{g j}=2\right)$ and that cell line $j$ is from component $1\left(z_{g j}=1\right)$. We choose component 1 to be the component with smaller mean so that negative $2 \mathrm{C}$ scores indicate decreased viability, or increased dependency in response to knockout. Conversely, positive $2 \mathrm{C}$ scores indicate decreased dependency in response to knockout. We assume that a minority of cell lines are responsive to gene knockout and thus refer to the component that contains fewer cell lines as the responsive component and the component with more cells lines as the background component. Summarizing this analysis, we say that differential dependencies whose responsive component has negative scores are increased dependencies, and those whose responsive component has positive scores are decreased dependencies.

Next, we construct the genomic alteration and cell-type feature matrix $A$. This matrix contains two parts. The first part of $A$ consists of genomic alterations. We use the OncoKB database [36] to select genes and mutations with annotated roles in cancer. For each cancer gene in OncoKB, we use the functional annotations of missense and nonsense somatic mutations to create three gene alteration features: missense activating mutations are combined into a single feature labeled GENE(A); missense and nonsense inactivating mutations are combined into a single feature labeled GENE(I); and the remaining unannotated missense mutations are combined into a single feature labeled GENE(0). The second part of $A$ contains cell-type features. In this analysis, we construct a binary feature for each cancer type represented in the analyzed cell lines. Each cancer type feature has the value 1 for cell lines of the corresponding cancer type and the value 0 for cell lines of other cancer types. We note that by definition, the cancer-type features are mutually exclusive across cell lines. Further details of both steps are in Methods

(2) Finding feature sets associated with differential dependencies. The second module in SuperDendrix is a rigorous and practically efficient combinatorial optimization algorithm to find sets $M$ of features (genomic alterations and/or cell-type features) in $A$ that are: (i) approximately mutually exclusive; and (ii) associated with increased (or decreased) dependency. We derive the SuperDendrix weight $W(M)$ of a set $M$ that combines criteria (i) and (ii), and use an integer linear program (ILP) to find the set $M^{*}$ of minimum (or maximum) weight $W\left(M^{*}\right)$ Methods,

(3) Evaluating statistical significance of associations. The third module of SuperDendrix includes two steps. First, a model selection step identifies a subset $\bar{M}$ of the features in $M^{*}$ found in the second module, where each feature in $\bar{M}$ contributes significantly to the weight $W(\bar{M})$. This step uses a conditional permutation test to iteratively remove features whose contribution to the weight $W\left(M^{*}\right)$ is nearly the same as random features. Second, we assess the statistical significance of the set $\bar{M}$ using a permutation test that conditions on both the number of cell lines that have each alterations and the number of alterations per cell line. Since the number of genomic alterations varies considerably across cell lines (Fig. S1), controlling for the number of alterations per cell line is important, as previously noted for predicting cancer driver mutations [37, 38].

We also developed an interactive tool for visualization and exploration of the SuperDendrix results which is available at: https://superdendrix-explorer.lrgr.io/, Further details of SuperDendrix as well as comparisons to another recent method [26] for mutual exclusivity analysis are in Methods. 


\section{Identification of differential dependencies and alteration features in DepMap}

We used SuperDendrix to analyze the Avana dataset [19Q1/2.19.2019] from Project DepMap containing results of CRISPR-Cas9 loss-of-function screens of 17,634 genes across 558 cancer cell lines from 31 cancer types [15, 39]. DepMap provides a dependency score for each gene knockout across all cell lines. These dependency scores are computed using the CERES algorithm [39], which is designed to reduce confounding at loci with high genomic copy number. CERES scores are scaled across all gene knockouts so that the median score for known "essential" genes is -1 and the median score for genes with "no dependency" is 0 . Following the " $6 \sigma$ " criterion of Tsherniak et al. [15], 1,730 genes have at least one cell line with a CERES score at least six standard deviations below or above the mean. We refer to CERES score profiles for these 1,730 genes as $6 \sigma$ differential dependencies (Table S1).

The first step of SuperDendrix computes that 492 (28\%) of the $6 \sigma$ differential dependencies are better fit by the two-component mixture model. We refer to these genes as two-component (2C) differential dependencies (Fig. 1b, Fig. S2, Table S2). The $4922 \mathrm{C}$ differential dependencies include 413 genes with increased dependency and 79 genes with decreased dependency. We find that 92 of the $2 \mathrm{C}$ differential dependencies are in the COSMIC Cancer Gene Census (CGC) [40] $(P<0.001)-$ including BRAF, KRAS, NRAS, and PIK3CA (Fig. S3 - a higher precision than for non-2C genes (2C: 18.7\%, non-2C: $11.0 \%$ ). These $2 \mathrm{C}$ genes are enriched $(\mathrm{FDR}<0.05)$ for $122 \mathrm{GO}$ molecular functions [41, 42] including kinase activity, G proteincoupled receptor activity, and DNA binding (Table S3). We find that the $6 \sigma$ differential dependencies that are not $2 \mathrm{C}$ differential dependencies either contain only a few outlier samples (e.g. $86 \%$ have fewer than 4 outlier samples) or have dependency score distribution that are unimodal with large variance (Fig. S4).

For the second input to SuperDendrix, we use 420,541 non-synonymous single-nucleotide mutations reported in sequencing data from the Cancer Cell Line Encyclopedia (CCLE) [43] for 554 of the 558 cell lines analyzed by DepMap. We also include the annotated cancer type of each cell line as a feature. For the feature selection step in the first module of SuperDendrix, we use mutation annotations from OncoKB [36] and obtain an alteration matrix of 566 alteration features in 363 genes with a total of 9,464 alterations across the 554 cell lines. Further details are in Methods.

\section{Associations between genomic alterations and differential dependencies}

We used SuperDendrix to identify associations between sets of genomic alterations and the $4922 \mathrm{C}$ differential dependencies. SuperDendrix identifies 23 single alterations and 9 sets of approximately mutually exclusive alterations that are significantly associated (FDR $\leq 0.2$ ) with differential dependencies (Fig. $2 \mathrm{a}$ and Table S4). 25 of these sets are associated with increased dependency and 7 with decreased dependency. Many of these associations are well-known dependencies including examples of oncogene addiction (e.g. $\mathrm{BRAF}(\mathrm{A})$ and increased dependency on $B R A F$ [47]) and synthetic lethality (e.g. ARID1A(I) and increased dependency on $A R I D I B[48]$ ). Half of the associations (16/32) group into three well-known cancer pathways (NFE2L2, RB1, and MAPK), and we highlight novel findings of SuperDendrix in these pathways.

First, SuperDendrix finds an association between the set $\{$ NFE2L2(A), KEAP1(I), KEAP1(0) $\}$ of three alterations and increased dependency on NFE2L2 (Fig. 2b). The KEAP1-NFE2L2 pathway is frequently perturbed in cancer with activating mutations in NFE2L2 or inactivating mutations in KEAP1 reported in more than $30 \%$ of lung squamous tumors [49, 50]. NFE2L2(A), KEAP1(I), or KEAP1(0) alterations occur in $51 / 554$ of the DepMap cell lines including 31\% (4/13) of lung squamous cancer cell lines. Moreover, three alterations are nearly mutually exclusive with only $2 / 51$ altered cell lines having more than one alteration (Fig. S5). Increased dependency on NFE2L2 in cell lines with NFE2L2(A) alterations is consistent with the oncogene addiction model [17, 18], since NFE2L2 is an oncogene in various cancers including lung, pancreas, breast, and gall bladder [50, 51]. The increased NFE2L2 dependency in cell lines with KEAP1 inactivating mutations is consistent with KEAP1's role in inhibiting NFE2L2 by targeting NFE2L2 for degra- 
bioRxiv preprint doi: https://doi.org/10.1101/2020.07.13.184697; this version posted July 14,2020 . The copyright holder for this preprint (which was not certified by peer review) is the author/funder, who has granted bioRxiv a license to display the preprint in perpetuity. It is made available under aCC-BY-NC-ND 4.0 International license.

a

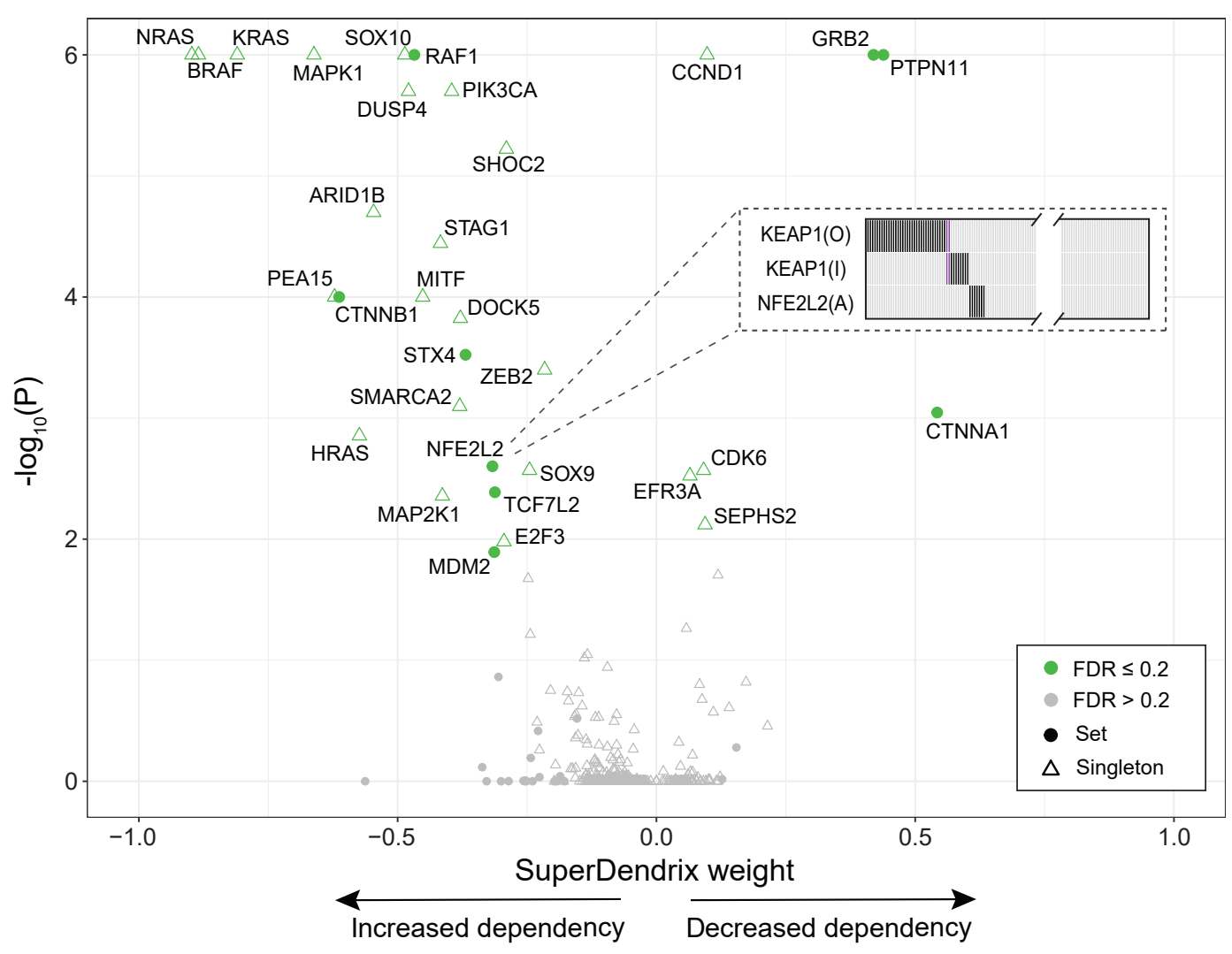

b

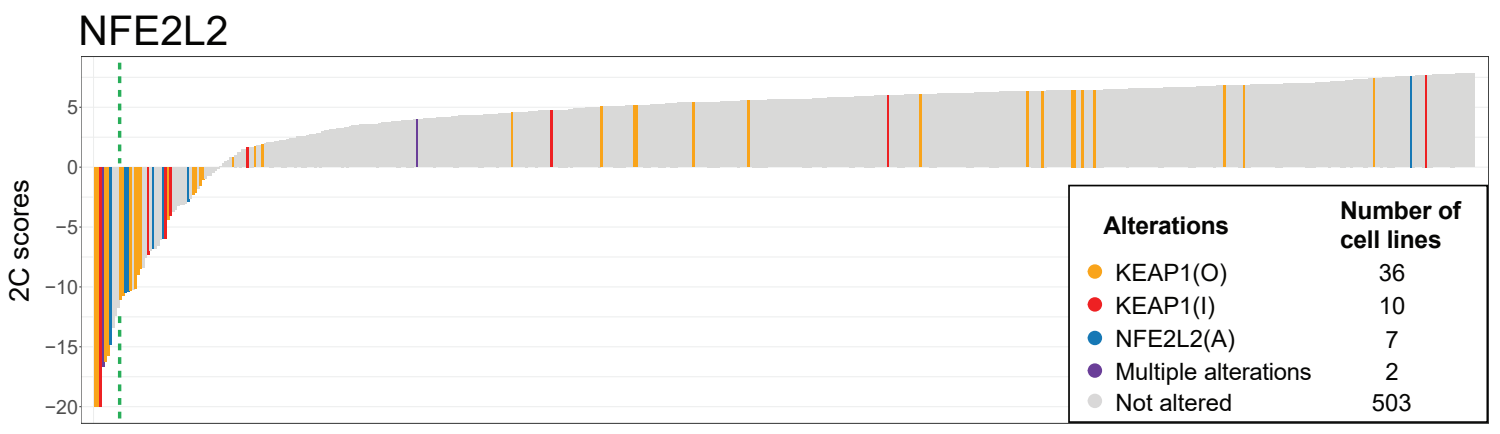

Cell lines

Increased dependency

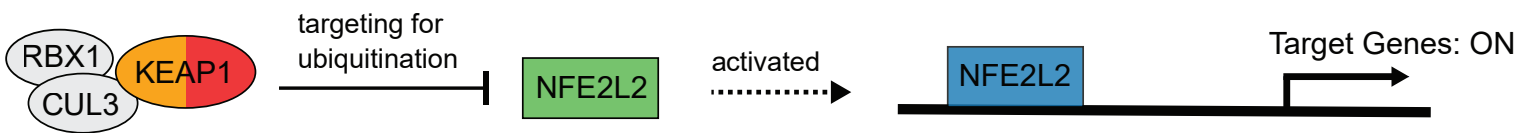

E3-ubiquitin

ligase complex

C

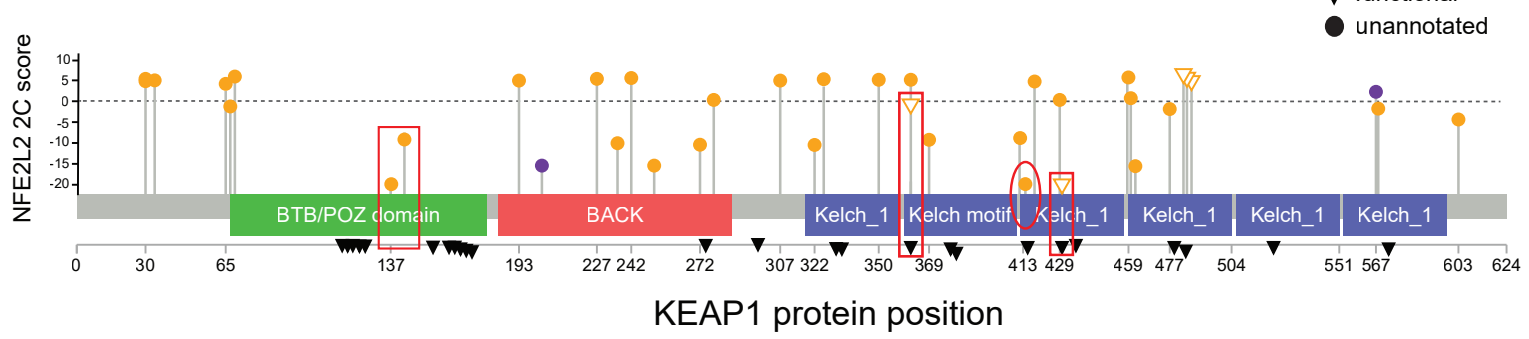


Figure 2: SuperDendrix identifies associations between genomic alterations and $2 \mathrm{C}$ differential dependencies in multiple biological pathways. (a) SuperDendrix weights and $P$-values for $262 \mathrm{C}$ differential dependencies with significant $(F D R \leq 0.2)$ associations with genomic alterations. 8 of these associations are sets of multiple alterations; e.g. the set $\{$ NFE2L2(A), KEAP1(I), KEAP1(0) $\}$ are alterations are (approximately) mutually exclusive (inset) and associated with increased dependency on NFE2L2. (b) (Top) Waterfall plot of 2C differential dependency scores for NFE2L2 across cell lines. Cell lines are colored by status in associated alteration set $\{$ NFE2L2(A), KEAP1(I), KEAP1(O)\}. Green dashed line indicates $6 \sigma$ threshold. (Bottom) KEAP1-NFE2L2 pathway. Solid circles are genes on the pathway, with colors indicating their alterations. Green boxes are genes that are knocked out. Association between KEAP1 inactivating mutations and increased dependency on NFE2L2 is consistent with the role of KEAP1 as an upstream activator of NFE2L2. (c) Locations of missense mutations in KEAP1 protein that are annotated as Other. KEAP1(0) mutations associated with increased dependency on NFE2L2 include: two mutations in the BTB/POZ domain (boxed) which is important for dimerization of KEAP1 [44], two annotated mutations in two of Kelch domains (boxed) which mediate interaction with NFE2L2 [45], and one mutation (circled) that lies at a residue that interfaces with NFE2L2 [38]. Orange (resp. purple) amino acid changes are in cell lines with exclusive (resp. multiple) mutations in KEAP1. Triangles indicate locations of mutations that are reported in Uniprot [46] to affect KEAP1-NFE2L2 interaction.

dation via ubiquitination. Inactivation of KEAP1 results in translocation of NFE2L2 to the nucleus where NFE2L2 targets over 200 genes for transcription [44, 52]. Thus, the increased dependency on NFE2L2 in cell lines with KEAPl inactivating mutations can be viewed as an another form of oncogene addiction, and NFE2L2 addiction in cell lines with somatic mutations in KEAP1 or NFE2L2 was recently reported [53]. Note that only a fraction of the associated alterations occur in cell lines whose CERES score is below the $6 \sigma$ threshold (Fig. 2 $\mathrm{b}$ ), demonstrating the advantages of SuperDendrix's $2 \mathrm{C}$ differential dependency score.

We find that associations with differential dependencies are also useful for annotating individual missense mutations. Several of the KEAP1(0) alterations - which include missense mutations that are unannotated in OncoKB - occur in cell lines with strong evidence of increased dependency on NFE2L2. These include 2 missense mutations in KEAP1 (G364C, G430V) that are not reported as functional in OncoKB, but occur at positions that are reported to disrupt NFE2L2 repression [45]. Another two mutations are in the BTB/POZ domain, a domain that is important for KEAP1 dimerization and KEAP1-CUL3 binding [44] (Fig. 22). Furthermore, the only KEAP1(0) mutations in the BTB/POZ domain are two mutations with negative $2 \mathrm{C}$ scores: the $21 \mathrm{KEAP} 1(0)$ with positive $2 \mathrm{C}$ scores mutations all lie outside the domain $(P=0.07$; binomial test). These findings prioritize these mutations for functional validation studies.

Second, SuperDendrix identifies associations between $R B 1$ inactivating mutations and differential dependencies in $C C N D 1, C D K 6$, and $E 2 F 3$, three members of the RB1 pathway (Fig. 3). Cell lines with $R B 1$ inactivating mutations have increased dependency on E2F3. It is known that active RB1 binds and inhibits E2F3 transcription factor activity, and that dissociation of the RB1-E2F3 complex results in E2F3initiated transcription of target genes that promote G1/S transition [54]. Our results suggest that cell lines with inactivating mutations in $R B 1$ become highly dependent on E2F3 activity, a phenomenon analogous to oncogene addiction [17, 18]. On the other hand, we observe that cell lines with $R B 1$ inactivating mutations are associated with decreased dependency on CCND1 and on CDK6. The CCND1-CDK4/6 complex is known to inactivate RB1 by phosphorylation. Thus, it makes sense that cell lines with inactive $R B 1$ do not require CCND1 or CDK6 to inactivate RB1, making these cell lines less sensitive to knockout of CCND1 and CDK6. This result suggests a correspondence between the direction of dependencies and the boolean logic in a pathway: there is increased dependency on an inhibitor of transcription factor $E 2 F 3$, but decreased dependency on inhibitors of an inhibitor of the transcription factor $E 2 F 3$. 


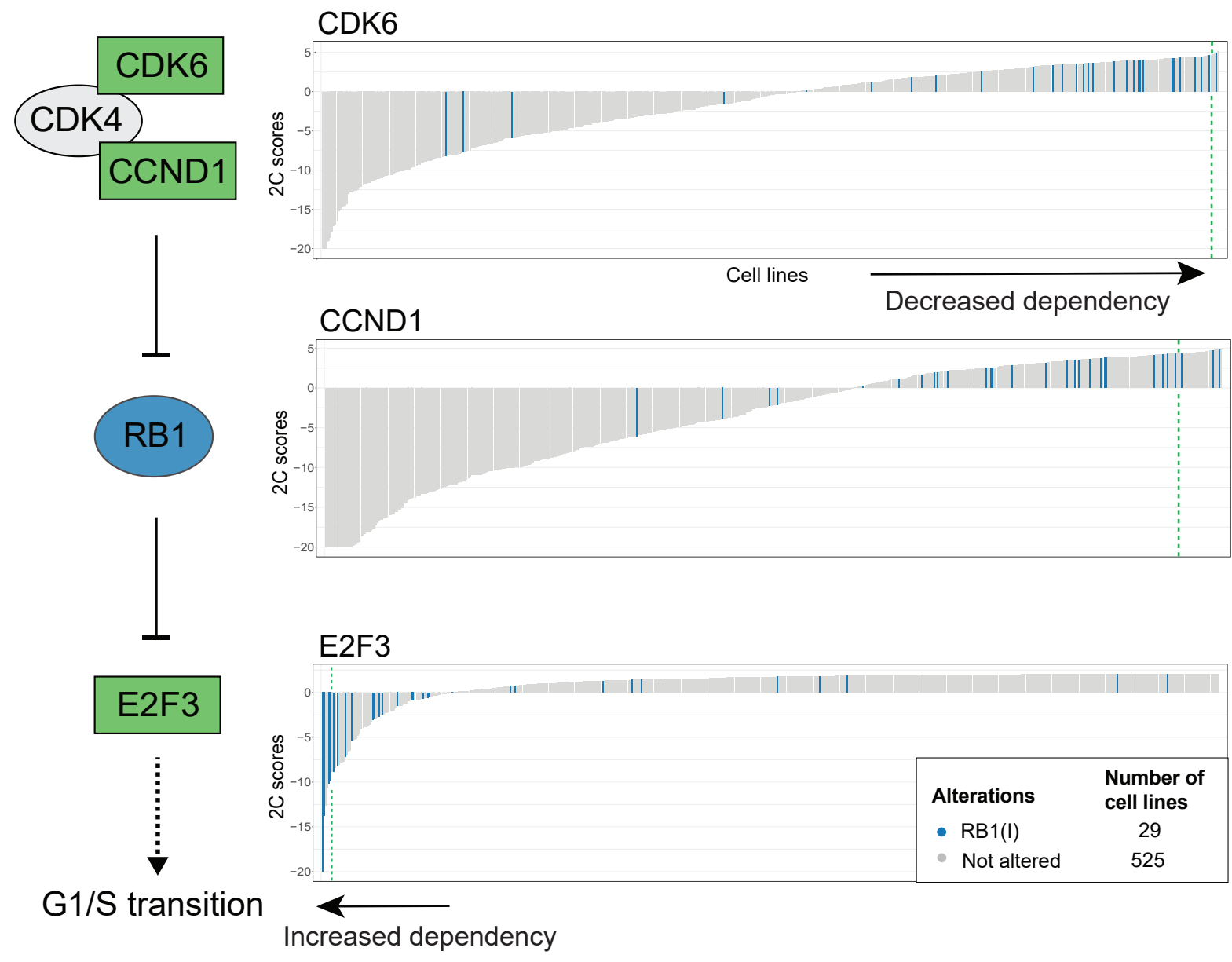

Figure 3: SuperDendrix identifies associations between genomic alterations and $2 \mathrm{C}$ differential dependencies in the RB1 pathway. $R B 1$ inactivating mutations are associated with increased dependency on $E 2 F 3$, consistent with RB1's role in inactivating the E2F3 transcription factor (Same format as Fig. 2p). On the other hand, $R B 1$ inactivating mutations are associated with decreased dependency on CDK6 and CCND1. This is consistent with the role of the CDK4/6-CCND1 complex in inactivating RB1. 


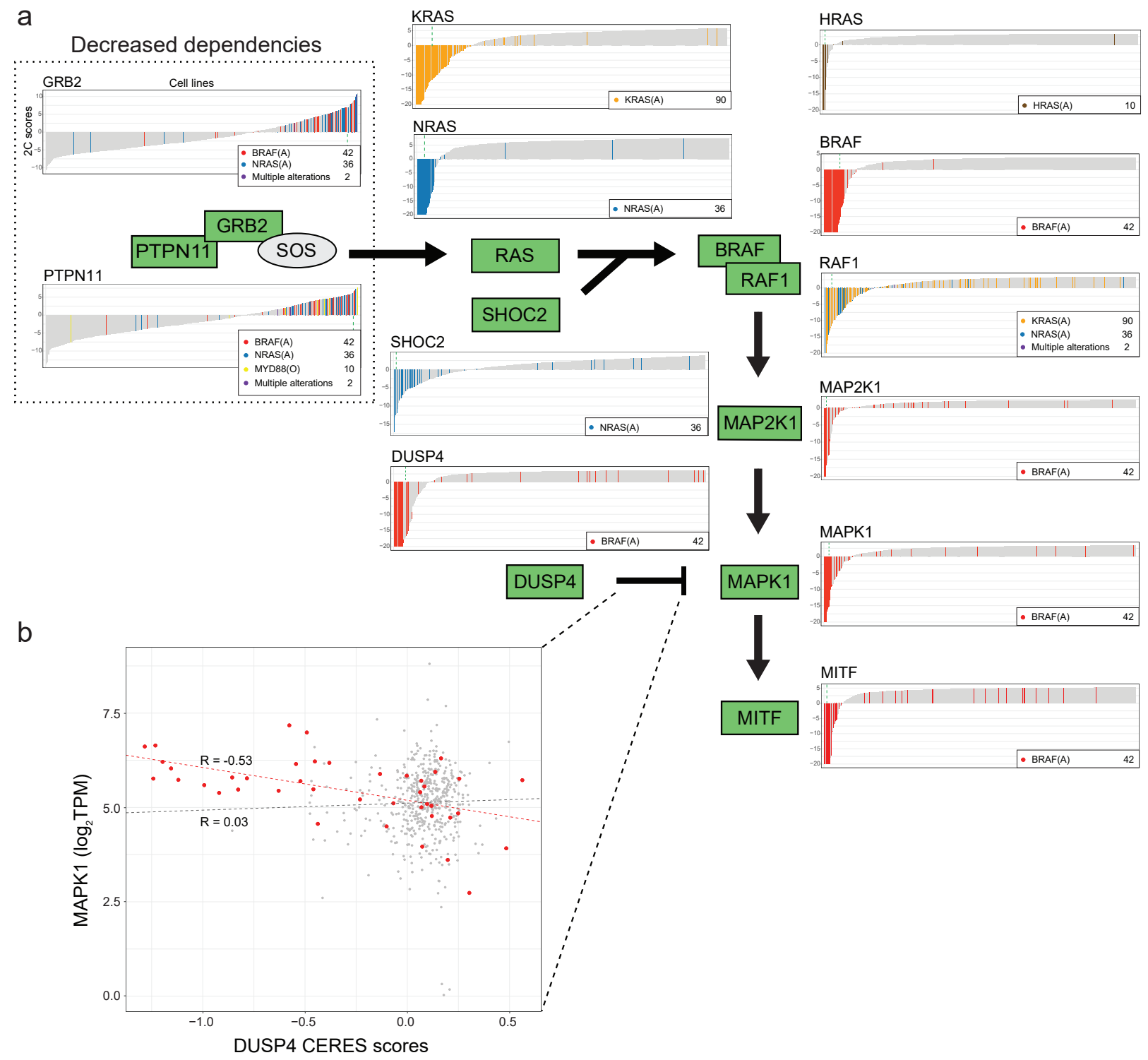

Figure 4: SuperDendrix identifies associations between genomic alterations and $2 \mathrm{C}$ differential dependencies in the MAPK pathway. (a) Activating mutations in $B R A F, K R A S, N R A S$, and $H R A S$, as well as other missense mutations in MYD88 are approximately mutually exclusive and associated with 12 differential dependencies in the MAPK pathway (Same format as Fig. 2p). Alterations that activate RAS/RAF are associated with increased dependencies of 10 downstream genes in pathway. In contrast, these same alterations are associated with decreased dependency on 2 genes, PTPN11 and GRB2, that are upstream activators of RAS. (b) CERES dependency scores of DUSP4 vs. expression of MAPK1. Cell lines with activating mutations in $B R A F$ (red dots) show negative correlation between DUSP4 dependency score and MAPK1 expression $(R=-0.53, P<0.001)$, while no correlation is observed in cell lines without $B R A F$ activating mutations $(R=0.03, P=0.499)$. 
Third, Superdendrix finds associations between 12 differential dependencies in the MAPK pathway and subsets of the approximately mutually exclusive alteration set $\{\operatorname{BRAF}(\mathrm{A}), \operatorname{KRAS}(\mathrm{A}), \operatorname{NRAS}(\mathrm{A}), \operatorname{HRAS}(\mathrm{A}), \operatorname{MYD} 88(0)\}$ (Fig. 4a). These include well-known associations between activating mutations in BRAF, KRAS, NRAS, or $H R A S$ and increased dependency on the corresponding gene [47, 55, 56, 57]. We also find an association between increased dependency on $R A F 1$ and the set $\{\operatorname{KRAS}(\mathrm{A}), \operatorname{NRAS}(\mathrm{A})\}$ of approximately mutually exclusive alterations. This is consistent with the role of RAF1 as a mediator of RAS for signal transduction in the MAPK pathway during transformation [58]. We also find an association between increased dependency on SHOC2 and NRAS(A) alterations as reported previously [59]. SuperDendrix also identifies associations between $\operatorname{BRAF}(A)$ alterations and increased dependency with other downstream members of the MAPK signaling pathway including MAP2K1, MAPK1, MITF, and DUSP4. Associations with MAP2K1, MAPK1, and MITF are consistent with previous reports on conditional dependency on these genes in BRAF(V600E) melanoma [60, 61, 62].

The association between $\operatorname{BRAF}(\mathrm{A})$ alterations and increased dependency on DUSP4 is intriguing because of conflicting reports on DUSP4's role in cancer. DUSP4 is reported to be a tumor suppressor that inhibits ERK1 and MAPK1 (ERK2) activity in the nucleus [63, 64]. As a tumor suppressor, DUSP4 knockout would be expected to result in decreased dependency. However, there are also reports of high expression of DUSP4 in colorectal cancers [63, 65] and skin cancers [66] with RAS or RAF alterations, suggesting that DUSP4 may contribute to oncogenesis in these cancers. Our finding that cell lines with $B R A F(A)$ have increased dependency on DUSP4 is consistent with an oncogenic role. Since DUSP4 is a negative regulator of MAPK1, we investigated the relationship between DUSP4 dependency and MAPK1. We found that in cell lines with $\mathrm{BRAF}(\mathrm{A})$, DUSP4 dependency scores were significantly negatively correlated $(R:-0.53, P \leq 0.001$; Pearson correlation) with expression of MAPK1 (Fig. 4b); i.e. cell lines with BRAF(A) and highest MAPK expression were the most dependent on DUSP4. In contrast, in cell lines without $\operatorname{BRAF}(\mathrm{A})$, there is no significant correlation between DUSP4 dependency and MAPK expression $(R: 0.03, P=0.499)$. These observations are consistent with the Goldilocks principle described in [67], where precise levels of biological factors must be maintained for strong fitness, with either overdose or lack of oncogenic signal resulting in regression of tumor. In this case, DUSP4 inhibition of MAPK1 is most essential in cell lines with hyperactive MAPK signaling due to BRAF(A) alterations.

SuperDendrix also identifies two associations between sets of alterations and decreased dependency on PTPN11 and GRB2 in the MAPK pathway. Specifically, we identify decreased dependency on PTPN11 in cell lines with $\operatorname{BRAF}(\mathrm{A}), \operatorname{NRAS}(\mathrm{A})$, or MYD88(0) alterations and decreased dependency on GRB2 in cell lines with $\operatorname{BRAF}(\mathrm{A})$ or $\operatorname{NRAS}(\mathrm{A})$ alterations. The decreased dependency on PTPN11 is consistent with a previous report that cell lines with constitutive RAS or RAF signaling were insensitive to suppression of PTPN11 [68]. Another study reports that MYD88, similar to RAF or RAS, activates MAPK during cell transformations [69], potentially explaining the association between MYD88(0) alteration and PTPN11 dependency exclusive of $\operatorname{BRAF}(\mathrm{A})$ and $\operatorname{NRAS}(\mathrm{A})$ alterations. Interestingly, 7 of the 10 MYD88(0) mutations occur in the TIR domain $(P=0.11$; binomial test) which is reported to modulate MYD88 activity via interaction with Toll and IL-1 receptors [70] (Fig. S6). While we are not aware of previous reports of associations with GRB2, it is intriguing that both proteins with decreased dependencies - PTPN11 and GRB2 - are upstream of the RAS/RAF mutations that result in constitutive MAPK signaling. Thus, it makes sense that cell lines with constitutive activation of RAS or RAF signaling are insensitive to upstream activators of RAS signaling, analogous with the insensitivity of RB1-deficient cell lines to knockout of upstream regulators CDK6 and $C C N D 1$ reported above (Fig. 3).

The remaining 16 associations beyond those in the three pathways described above include associations between members of the same protein complex, and associations in other cancer-implicated pathways. Associations in protein complexes include: increased dependency on ARIDIB in cell lines with ARID1A(I) alteration [48], increased dependency on SMARCA2 in cell lines with SMARCA4(I) alteration [71], and increased dependency on STAG1 in cell lines with STAG2(I) alteration [72]. Notable associations in pathways 
include 2 associations in the Wnt pathway: increased dependency on CTNNB1 in cell lines with APC(I) or CTNNB1(A) alterations and increased dependency on TCF7L2 in cell lines with APC(I) or CTNNB1(A) alterations (see 'Associations in Wnt pathway' in Supplementary Information).

We found that 22 of the 32 associations identified by SuperDendrix validated in the Score dataset of CRISPR screens from Behan et al. [16], where we consider an association to be validated if there is a significant difference ( $P \leq 0.05$; Wilcoxon rank sum test) in dependency scores between cell lines containing associated mutations and those without such mutations (Table S4). Many of the associations that did not validate were in cancer types with few cell lines in the Score dataset. For example, several associations with $\mathrm{BRAF}(\mathrm{A})$ did not validate in the Score dataset; however, this is not surprising since the majority of $\mathrm{BRAF}(\mathrm{A})$ mutations in the Avana dataset are in the 34 skin cancer cell lines, while the Score dataset contains only 4 skin cancer cell lines (Table S5]. Further details are in sections 'Comparison to other perturbation screen results' and 'Validation on the Sanger CRISPR-Cas9 screen data' in Supplementary Information.

Finally, we compared the associations between mutations and dependencies identified by SuperDendrix to those identified by Tsherniak et al. [15] in RNAi data and by Behan et al. [16] in a different CRISPR dataset. SuperDendrix finds significant associations for $6.5 \%$ of differential dependencies. This is higher than $1.3 \%$ in Tsherniak et al. and $1.6 \%$ in Behan et al.

\section{Cancer-type-specific differential dependencies}

Next, we investigated associations between differential dependencies and cancer type. We augmented the alteration matrix with 31 cancer-type features, each feature representing one of the 31 cancer types in the Avana dataset. We ran SuperDendrix on the augmented alteration matrix and identified 135 differential dependencies that are significantly associated (FDR $\leq 0.2)$ with alterations and/or cancer types (Table S6). These include the 32 differential dependencies identified above using SuperDendrix on alterations alone. 18 associations are identical to those found by SuperDendrix when run on alterations alone while the remaining 117 include at least one cancer type feature. Among these are 14/32 profiles that were identified in the SuperDendrix analysis of genomic alterations described above but with associated sets that include cancer types and result in higher SuperDendrix weights. For example, MITF dependency and BRAF(A) (SuperDendrix weight $=-0.21)$ has stronger association with Skin cancer (SuperDendrix weight $=-0.75)$.

Of the 117 associations that include a cancer type feature, 92 are associations with increased dependency upon gene knockout, with the remaining 25 associated with decreased dependency. These 117 differential dependencies are enriched (FDR $\leq 0.05$ ) for $85 \mathrm{GO}$ molecular function terms (Table $\mathrm{S} 7 \mathrm{p}$. The most significant function in these terms is regulatory region nucleic acid binding: in particular, 41 of the 117 are annotated as human transcription factors in [73] (fold enrichment $=4.07, P \leq 0.001$ ), a greater enrichment than the 70 transcription factors found among all 492 differential dependencies (fold enrichment $=1.65$, $P \leq 0.001$ ). This enrichment of transcription factor dependencies is consistent with previous reports; e.g., [15] identified 49 transcription factors with strong lineage-specific dependencies from RNAi screens. Our results include 15 of these 49 as well as 26 additional transcription factor dependencies that were not reported in [15].

The 41 transcription factor dependencies with cancer-type-specific associations cluster into a number of interesting groups (Fig. 5). These include dependencies on ISL1, HAND2, and MYCN in neuroblastoma, all of which were recently reported as part of the core regulatory circuitry (CRC) in neuroblastoma and associated with superenhancers [74]. Other large classes of cancer-type dependencies are in skin cancer (8 dependencies), breast cancer (5), leukemia or lymphoma (13), and multiple myeloma (10).

Two prominent cancer-type-specific associations identified by SuperDendrix are in the ID3-TCF3-CCND3 pathway in blood cancers (Fig. 6). Specifically, SuperDendrix finds an association between increased dependency on the transcription factor TCF3 and the mutually exclusive set \{multiple myeloma, ID3(I), MEF2B(A)\}, as well as an association between increased dependency on CCND3 and a mutually exclusive set $\{$ leukemia, 


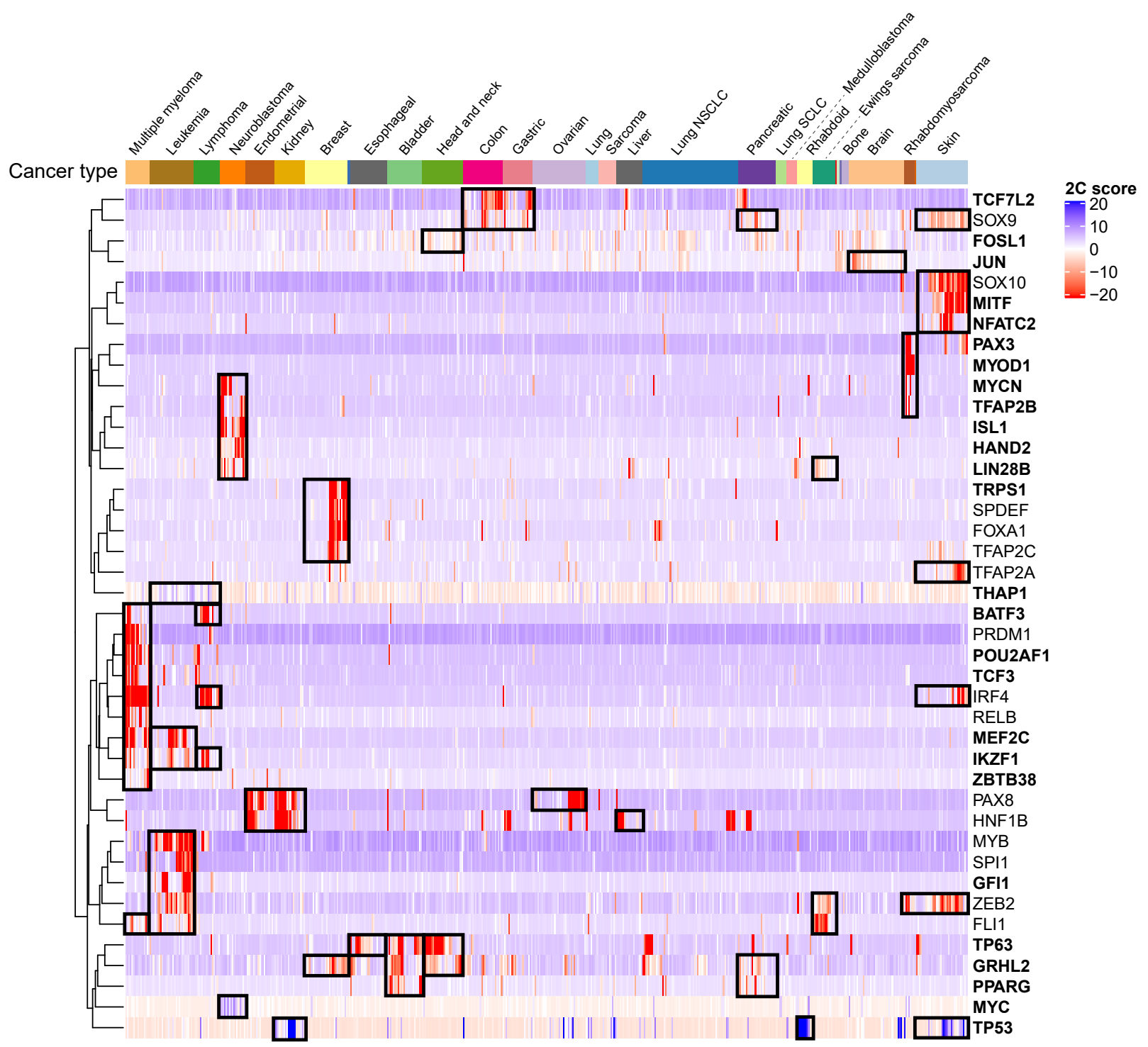

Figure 5: Transcription factors are the most common class of cancer-type-specific differential dependencies. Heatmap of $2 \mathrm{C}$ scores for 41 transcription factors that SuperDendrix identifies as cancer-typespecific differential dependencies. Dependency profiles are clustered within and across cancer types, with black boxes highlighting groups of prominent dependencies across cancer types. Bold text indicates transcription factors that were not reported in RNAi analysis [15]. Labels are shown for cancer types with at least 5 cell lines. 

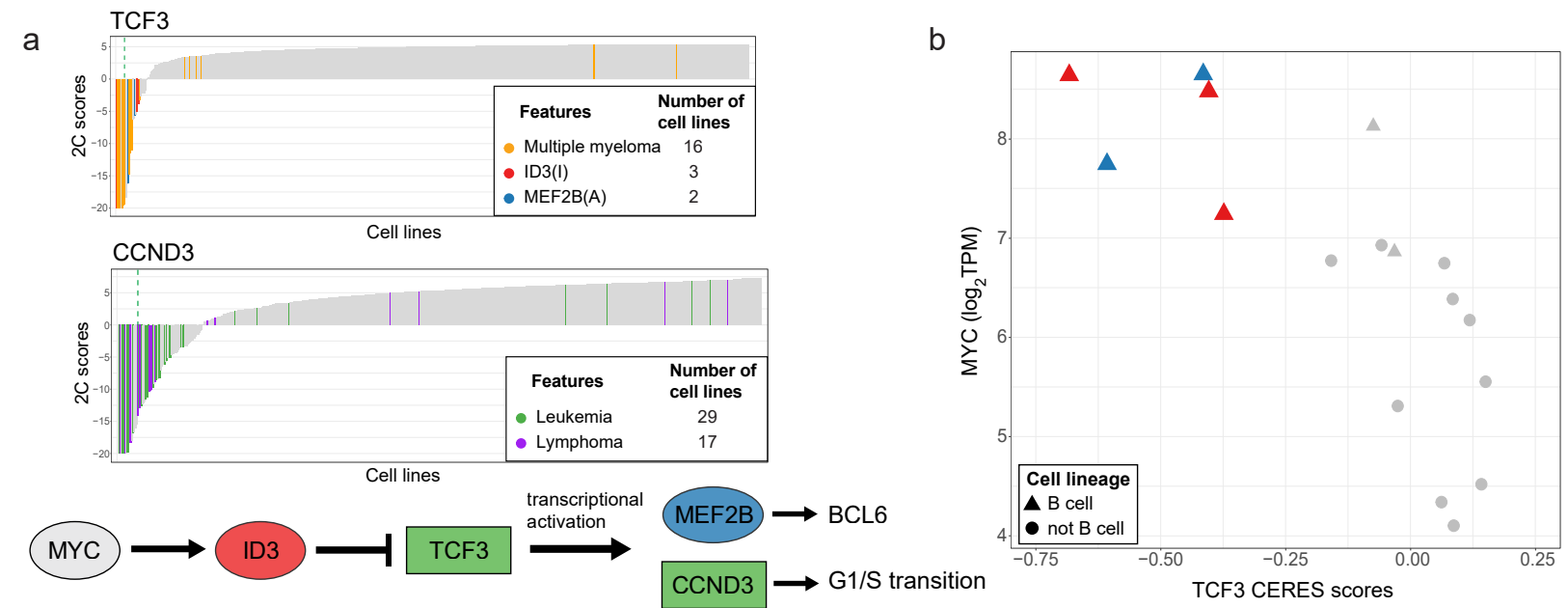

Figure 6: TCF3 and CCND3 dependencies in blood cancers (a) (Top) SuperDendrix identified cancer-type-specific associations between increased dependency on TCF3 and a mutually exclusive set \{multiple myeloma, ID3(I), MEF2B(A)\} and also between increased dependency on CCND3 and a mutually exclusive set \{leukemia, lymphoma\}. (Bottom) The ID3-TCF3-CCND3 pathway (Same format as Fig. 2b). (b) TCF3 CERES scores in lymphoma cell lines vs. expression of MYC. The 5 cell lines with ID3 or $M E F 2 B$ alterations are among cell lines with lowest TCF3 CERES scores and highest MYC expression, and include 5 of $7 \mathrm{~B}$-cell lymphoma cell lines in dataset.

lymphoma (Fig. 6a). Chromosomal translocations resulting in TCF3 fusion genes occur in various leukemias and lymphomas with differing reports regarding the importance of activation vs. inactivation of TCF3 in oncogenesis [75, 76]. The association between increased dependency on TCF3 and ID3(I) or MEF2B(A) alterations is consistent with reports that ID3 inhibits TCF3 [77], and that TCF3 binds upstream of $M E F 2 B$ [78, 79]. Thus, this association conforms to the model of oncogenic pathway addiction, with increased dependency on TCF3 in cell lines with inactivating mutations in the TCF3-inhibitor ID3 (analogous to the associations described above with the inhibitors KEAPl and $R B 1$ ) or in cell lines with activating mutations in the downstream transcriptional activator $M E F 2 B$. TCF3 has previously been suggested as a dependency in multiple myeloma cell lines, promoting tumorigenesis in cooperation with MYC [80]. In addition, the ID3-TCF3-CCND3 pathway is reported to be frequently perturbed in B-cell lymphoma with elevated MYC expression due to a chromosomal translocation [77]. Interestingly, the 5 cell lines with ID3(I) or MEF2B(A) mutations are 5 of the 7 B-cell lymphomas in the data set; moreover, the two MEF2B(A) alterations in these cell lines are D83V amino acid changes that are reported to be relevant in development of lymphoma [81]. Consistent with these reports, we found that the $5 \mathrm{~B}$-cell lymphoma cell lines with increased dependency on TCF3 and ID3(I) or MEF2B(A) alterations have high MYC expression (Fig. 6b). Interestingly, we find that the increased dependencies on $T C F 3$ and $C C N D 3$ are approximately mutually exclusive, and the cell lines with both dependencies are the subset of B-cell lymphoma cell lines containing either ID3(I) or MEF2B(A) mutations (Fig S7). Taken together, these results show increased dependency on the ID3-TCF3-CCND3 pathway in multiple myeloma, leukemia, and lymphoma, with the association in B-cell lymphomas modulated through alterations in $I D 3$ or $M E F 2 B$.

The other 76 cancer-type-specific differential dependencies are enriched (FDR $\leq 0.05$ ) for $46 \mathrm{GO}$ molecular function terms (Table S8), with the top 3 enriched terms being enzyme binding (GO:0019899), catalytic activity (GO:0003824), and kinase activity (GO:0016301). These 76 differential dependencies include genes known to be overexpressed or predictive of prognosis for the associated cancer type such as increased dependencies of $M E T$ in brain cancer [82] and $L D B 1$ and $L M O 2$ dependencies in leukemia 
a
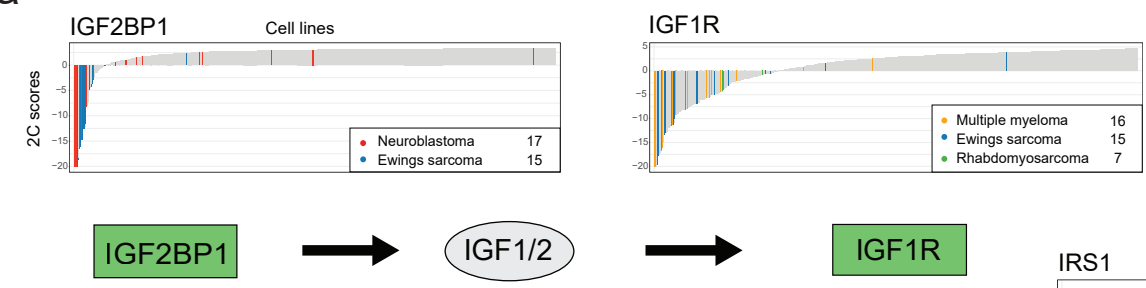

b
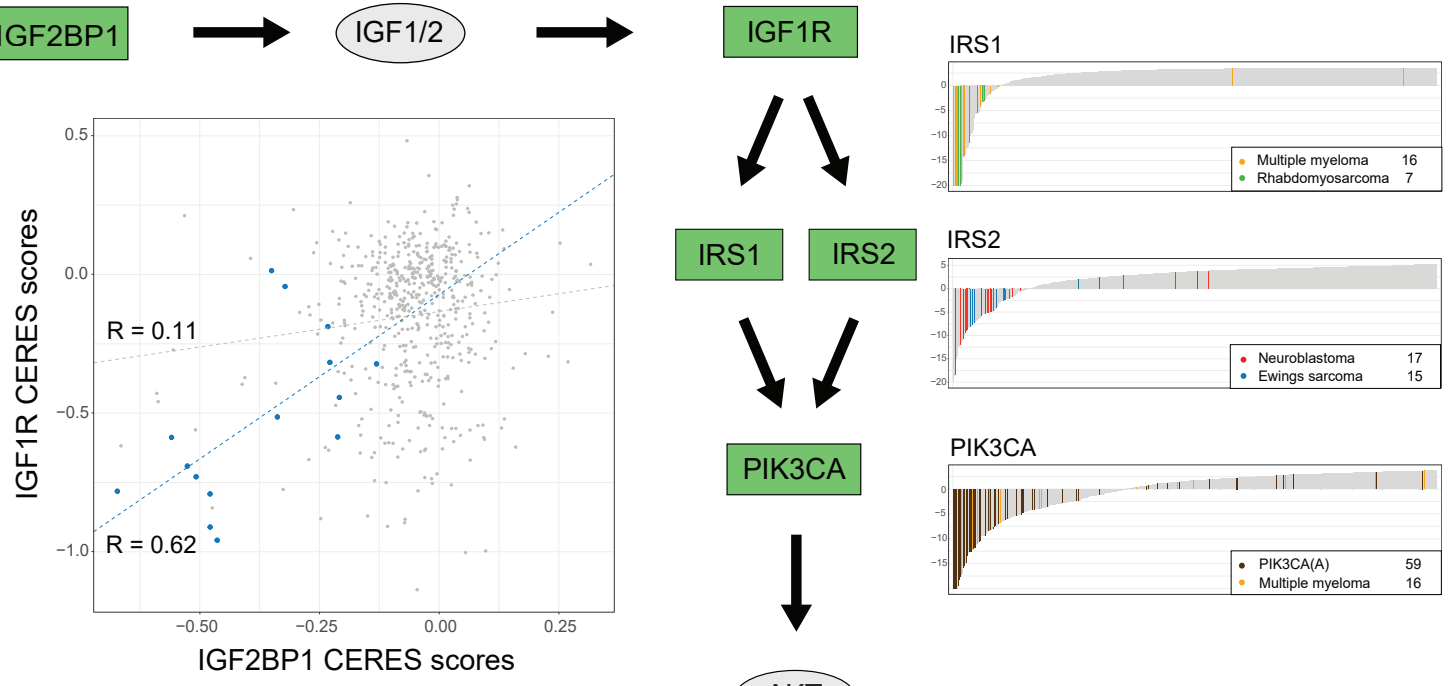

C
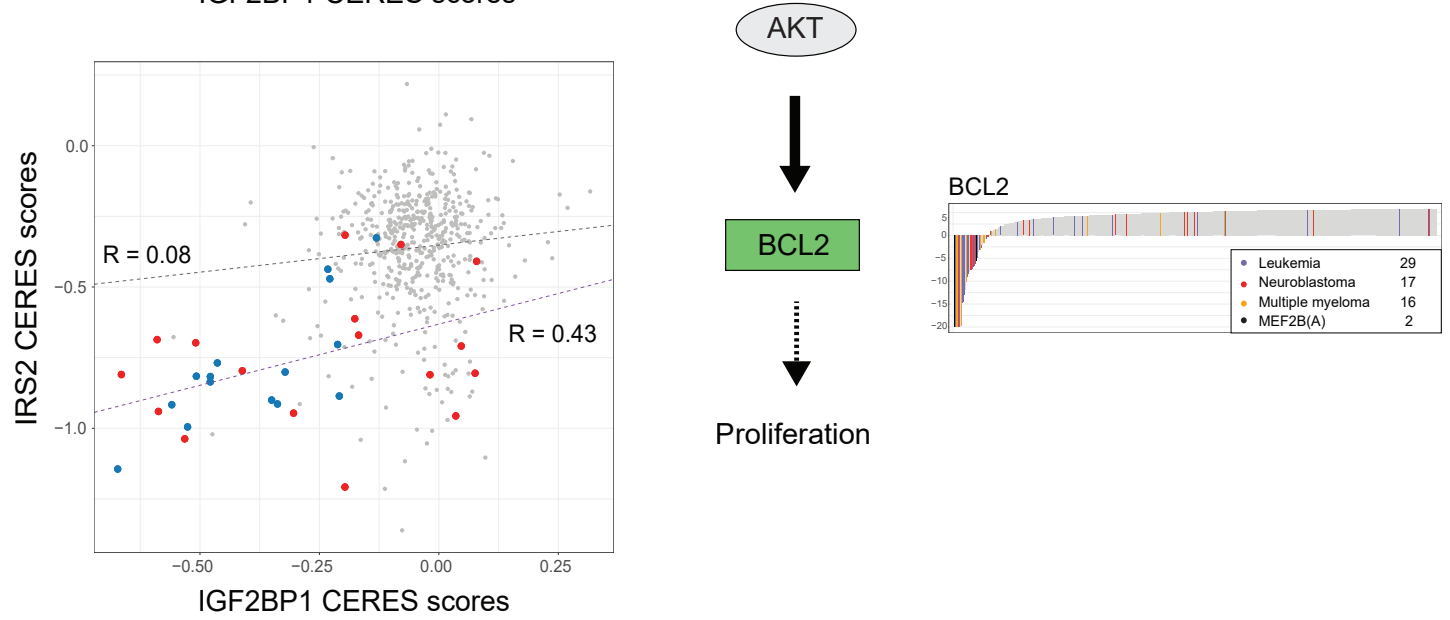

Proliferation

Figure 7: Cancer-type-specific differential dependencies in the IGF1R/PI3K pathway. (a) SuperDendrix identifies cancer-type-specific dependencies between 6 genes in IGF1R/PI3K pathway across multiple cancer types (Same format as Fig. 22p). (b) CERES scores of IGF2BPI and IGFIR are positively correlated ( $R=0.62$ ) in Ewing's sarcoma cell lines (blue points), but only weakly correlated $(R=0.11$ ) across other cancer types (gray points). (c) CERES scores of IGF2BPI and IRS2 are positively correlated ( $R=0.43)$ in Ewing's sarcoma (blue points) and neuroblastoma (red points) cell lines, but only weakly correlated $(R=0.08)$ across other cancer types (gray points). 
[83, 84]. Several additional associations correspond to dependencies on upstream regulators of cancer genes such as MDM2 in skin and kidney cancers and EGFR in head and neck cancer.

A prominent grouping of cancer-type-specific differential dependencies are 6 genes in the IGF1R and PI3K pathways (Fig. 79) across several cancer types. In the IGF1R pathway, we find increased dependency on IGF2BP1, IGFIR, IRS1 or IRS2 in neuroblastoma, Ewing's sarcoma, multiple myleoma, and rhabdomyosarcoma. These dependencies are consistent with previous reports of dependencies on IGF1R in Ewing's sarcoma and rhabdomyosarcoma [85]. SuperDendrix also find increased dependencies on PIK3CA and $B C L 2$ in some of these same cancer types, including PIK3CA in multiple myeloma and BCL2 in leukemia, multiple myeloma, and neuroblastoma [86, 87]. These findings are consistent with IRS1/2's role in activating the PI3K pathway [88]. Since dysregulation of the PI3K pathway results in tumor proliferation [89], all of these increased dependencies are consistent with a phenotype of oncogenic pathway addiction in the IGF1R/PI3K pathway.

Since neuroblastoma, Ewing's sarcoma, multiple myleoma, and rhabdomyosarcoma cell lines each exhibited dependencies on more than one gene in the IGF1R pathway, we examined whether individual cell lines harbored multiple dependencies. We found strong cancer-type-specific correlations between dependency scores of pairs of genes in the IGF1R pathway. These include correlations between IGF2BPI and $\operatorname{IGFIR}(R=0.62, P<0.001)$ in Ewing's sarcoma (Fig.7p) and between IGF2BP1 and IRS2 dependencies ( $R=0.43, P<0.001$ ) in Ewing's sarcoma and neuroblastoma (Fig. 77). Importantly, these correlations are weaker in other cancer types ( $R=0.11$ and $R=0.08$, respectively), and consequently were not reported in two recent studies [22, 90] that examined correlations between dependency profiles across all cell lines in DepMap. In addition, many of the cell lines with these correlated dependencies have CERES scores larger than the -0.6 threshold used to define dependency in DepMap [39]. Thus, the identification of these correlations relied on both SuperDendrix's 2C scores and SuperDendrix's ability to identify cancer-type-specific associations. At the same time, we find strong correlations between IGFIR with IRSI and IGFIR with $I R S 2$ across all cell lines, as previously reported in [22, 90]. We also find cancer-type-specific correlations between $C H U K$ and $I K B K B$ dependencies in lymphoma and multiple myeloma (Fig. S8).

Lastly, we found that 72 of the 117 associations identified by SuperDendrix are validated in the Score dataset [16], using the same test as described above (Table S6. Also as above, many of the associations that did not validate were in cancer types that were not well represented in the Score dataset including multiple myeloma, skin, and rhabdomyosarcoma (Table S5). Further details are in 'Validation on the Sanger CRISPR-Cas9 screen data' in Supplementary Information.

\section{Discussion}

We introduced SuperDendrix, a method that incorporates a principled statistical model and a practically efficient combinatorial algorithm to identify associations between differential dependencies and genomic alterations or cancer types. SuperDendrix scores differential dependencies using a two-component mixture model and identifies mutually exclusive sets of features - including genomic alterations and/or cell types - that are associated with each differential dependency. Application of SuperDendrix to data from Project DepMap - the largest publicly dataset of CRISPR-Cas9 loss-of-function screens in hundreds of cancer cell lines - identified 492 differential dependencies and inferred associations between 135 (27.4\%) of these dependencies and sets of genomic alterations and/or cancer types. Many of these associations group into well-known cancer pathways such as MAPK, RB1, and IGF1R. SuperDendrix finds a higher fraction of differential dependencies are associated with genomic alterations compared to previous analyses of RNAi and CRISPR screens [15, 16, 24]. This illustrates advantages of the SuperDendrix method, including more stringent selection of differential dependencies and the search for sets of associated biomarkers; in contrast, existing approaches have very permissive definitions of differential dependencies or restrict to evaluating 
single-biomarker associations.

Interestingly, we observe striking consistencies between the directionality of dependencies (increased vs. decreased), the type of interactions (activating vs. inhibitory), and the position of dependencies and genomic alterations in pathways. Oncogenic mutations in upstream pathway genes - such as activating alterations in an oncogene or inactivating alterations in an tumor suppressor - are associated with increased dependencies in genes that are downstream in the same pathway and that promote cancer; e.g. NFE2L2 dependency in cell lines with inactivating mutations in KEAPI and MAPKI dependency in cell lines with activating mutations in $B R A F$ ). These results are consistent with the notion that cancer cells develop addiction to an oncogenic pathway during cancer progression [17, 18]. On the other hand, oncogenic alterations in downstream pathway genes - such as activating alterations in an oncogene or inactivating alterations in a tumor suppressor gene - are associated with decreased dependencies on upstream genes of the same pathway; e.g. cell lines with inactivating mutations in $R B 1$ show decreased dependency on CDK6. These results show the importance of considering pathway topology in the design of cancer therapeutic strategies; for example, a current strategy for treating tumors with activating mutations in undruggable oncogenes is to inactivate downstream genes [91]. At the same time, current annotations of interactions in pathways should be interpreted with care and potentially revised with knowledge gained from perturbation experiments. For example, DUSP4 is noted as a tumor suppressor due to its role in inhibiting MAPK signaling; however, we find increased dependency on DUSP4 in cell lines with activating mutations in BRAF suggesting that DUSP4 contributes to maintaining the balance of MAPK signaling in BRAF mutant tumors. These results suggest DUSP4 as a potential therapeutic target for cancer treatment. Our results also provide further predictions about the the functional consequences of individual non-synonymous mutations and the function of individual genes. For example, we find that previously unannotated mutations in the dimerization domain of KEAP1 are associated with increased dependency on its downstream target, NFE2L2.

SuperDendrix also identifies associations between differential dependencies and sets of cancer types or combinations of cancer types and genomic alterations. A large fraction (35\%) of the cancer-type specific associations found by SuperDendrix involve increased dependencies on lineage-specific transcription factors. Many of these lineage-specific transcription factors have been previously reported to be highly expressed or correlated with poor prognosis in cancers of corresponding types. We also identify associations that include both cancer types and genomic alterations. For example, we find that increased dependency on TCF3 in multiple myeloma cell lines or in cell lines with $I D 3$ or $M E F 2 B$ alterations. These genomic alterations occur in the majority of B-cell lymphoma cell lines in the dataset. Another prominent cancer-type-specific association found by SuperDendrix is increased dependency on $I G F 2 B P 1$, a regulator of insulin growth factor receptor $I G F 1 R$, in Ewing's sarcoma and neuroblastoma. We anticipate that with larger cohorts, there will be increased opportunities to identify these more subtle associations that include both cancer types and genomic alterations.

There are a number of directions for future work, both the analysis and in further development of SuperDendrix. First, our current analysis does not include copy number aberrations (CNA) and DNA methylation changes, both of which are likely to be associated with differential dependencies. Since these alterations span larger genomic distances than single-nucleotide mutations, they require more careful decomposition into specific alteration events [92]. Since CNA occur frequently in solid tumors, we may be underestimating the number of associations in these cancer types. Second, alternative dependency scores could be used as input to SuperDendrix; recent studies have demonstrated that CERES scores can be affected by other covariates and confounding variables [93, 94]. Third, we found that some differential dependencies are associated with multiple sets of features (e.g. increased dependency on TCF7L2 and the sets $\{$ APC(I), CTNNB1(A) $\}$ and $\{$ Colon, Gastric $\}$ ). Extending SuperDendrix to simultaneously identify multiple sets of features might identify additional such dependencies, as previously shown for multiple sets of mutually exclusive mutations [95]. Finally, SuperDendrix is a general algorithm that can be used to find associations between binary features (e.g. germline or somatic mutations, cell types) and quantitative phenotypes (e.g. drug response, 
bioRxiv preprint doi: https://doi.org/10.1101/2020.07.13.184697; this version posted July 14,2020 . The copyright holder for this preprint (which

was not certified by peer review) is the author/funder, who has granted bioRxiv a license to display the preprint in perpetuity. It is made available under aCC-BY-NC-ND 4.0 International license.

cell size). It would be interesting to analyze these other phenotypes using SuperDendrix, particularly drug response data from The Genomics of Drug Sensitivity in Cancer (GDSC) database [96], and compare against other methods [97, 98] that have been designed specifically to identify associations between drug response and genomic features. 


\section{Methods}

\section{SuperDendrix algorithm}

We introduce a new algorithm, SuperDendrix, to identify sets of binary features (e.g. genomic alterations or cell types) that are approximately mutually exclusive and associated with a continuous-valued phenotype. The inputs to SuperDendrix are:

1. An $l \times n$ matrix $P=\left[p_{g j}\right]$ of $l$ quantitative phenotypes measured in $n$ samples. Each entry $p_{g j}$ is the score of phenotype $g$ in sample $j$. Each row of the phenotype matrix corresponds to a phenotype profile.

2. A list of binary features (e.g. somatic alterations) for each sample.

3. (Optional) Categorical information (e.g. cell type) of each sample.

While SuperDendrix is a general-purpose algorithm, here we describe the specific application where the phenotype scores are dependency scores from gene perturbation experiments and the binary features are genomic alterations (and optionally cell types). SuperDendrix includes three modules: (1) A module to identify and score differential dependencies using a two-component mixture model and to select genomic and cell-type features using mutation annotations; (2) A module to find sets of alterations/features that are approximately mutually exclusive and associated with differential dependencies using a combinatorial optimization algorithm; (3) A module to perform model selection and to evaluate statistical significance of associations.

\section{Identifying differential dependencies and selecting genomic features}

The first module in SuperDendrix includes two steps: the identification and scoring of differential dependencies and the selection of genomic and cell-type features. In the first step, we assume that a gene perturbation leads to two population of samples: a minority of samples are responsive to the perturbation while the remaining samples are unresponsive and have scores derived from a background distribution. Thus, we assume that the dependency scores are distributed according to a two-component mixture model. We fit each dependency profile with a $t$-distribution and with a mixture of two $t$-distributions, using the $t$-distribution to model high variance in the dependency scores [99]. We use the Bayesian information criterion (BIC) [100] to select between the one-component or two-component models; we refer to genes whose dependency profiles are better fit by a two-component mixture as differential dependencies.

For each differential dependency $g$ and sample $j$, we compute the 2C score, or differential dependency score, $d_{g j}=\frac{\operatorname{Pr}\left(z_{g j}=2 \mid p_{g j}\right)}{\operatorname{Pr}\left(z_{g j}=1 \mid p_{g j}\right)}$, the $\log$ ratio of the posterior probabilities that the observed score is from component 2 or component 1 . We compute posterior probabilities by fitting the dependency scores to a mixture of two Gaussian distributions. We choose component 1 to be the component with smaller mean so that negative $2 \mathrm{C}$ scores indicate decreased viability, or increased dependency in response to knockout. Conversely, positive $2 \mathrm{C}$ scores indicate decreased dependency in response to knockout. We assume that a minority of cell lines are responsive to gene knockout and thus refer to the component that contains fewer cell lines as the responsive component and the component with more cells lines as the background component. We define the $2 \mathrm{C}$ profile, or differential dependency profile, $d_{g}=\left(d_{g 1}, \ldots, d_{g n}\right)$ to be the differential dependency scores across all samples. Profile $d_{g}$ is an increased dependency if its responsive component contains cell lines with negative $2 \mathrm{C}$ scores (increased dependency) and a decreased dependency if its responsive component contains cell lines with positive $2 \mathrm{C}$ scores (decreased dependency).

In the second step, we construct a genomic alteration matrix $A$ that includes annotated genomic alterations and (optionally) cell-type features. We construct $A$ from non-synonymous somatic mutations in 
cancer genes in the OncoKB database [36]. We first classify each gene in OncoKB as Oncogene, Tumor suppressor, or Other based on "Oncogenicity" and "Alteration" columns in the OncoKB annotation as follows. For each gene, we select mutations with the following oncogenicity: "Likely Oncogenic," "Oncogenic," or "Inconclusive." We classify genes that include "Deletion" or "Truncating Mutations" in the Alteration column as Tumor suppressor genes. We classify genes containing only "Inconclusive" mutations as Other genes. Finally, we classify each of the remaining genes as Oncogene. For each gene GENE in OncoKB, we group mutations from the input list into Activating (oncogene), Inactivating (tumor suppressor), or Other alteration features which we label as GENE(A), GENE(I), and GENE(O) using the OncoKB annotation according to the following rules:

1. If GENE is Oncogene, its missense mutations that are included in the list of amino acid changes in OncoKB are grouped into a feature, GENE(A). Mutations in GENE that are not annotated in OncoKB are grouped into a feature, $\operatorname{GENE}(\mathrm{O})$.

2. If GENE is Tumor suppressor, its missense mutations that are included in the list of amino acid changes in OncoKB and mutations annotated as \{nonsense, nonstop, frameshift, start codon, stop codon, splice site, de novo start out of frame $\}$ are grouped into a feature, GENE(I). Mutations in GENE that are not annotated in OncoKB are grouped into a feature, GENE(O).

3. If GENE is Other, then mutations are grouped into a feature, $\operatorname{GENE}(\mathrm{O})$.

Using the OncoKB alteration features derived above, we construct a genomic alteration matrix $A=\left[a_{i j}\right]$ of $m$ OncoKB alteration features across $n$ samples where $a_{i j}=1$ if alteration $i$ occurs in sample $j$ and $a_{i j}=0$ otherwise.

Next, we generate binary features that represent the cell type of each sample using information from metadata such as the primary tissue. In the application in this paper, we use cancer types as the cell-type features. Each cancer-type feature is marked as 1 for samples of that type, and as 0 for samples of other types. Note that the cancer-type features are mutually exclusive by definition. We now combine the two sets of features and create an augmented binary feature matrix $A$ of $m$ OncoKB alteration features and $q$ cancer-type features across $n$ samples.

\section{Finding feature sets associated with differential dependencies}

The second module in SuperDendrix finds a subset $M^{*}$ of features (rows in $A$ ) that are: (i) most associated with differential dependency profile $d_{g}$; and (ii) approximately mutually exclusive.

First, for each score $d_{g j}$ of differential dependency $g$ in sample $j$ from the profile $d_{g}$, we define a normalized score $d_{j}^{\prime}=\frac{d_{g j}}{S}$ where $S=\sum_{d_{g j}<0}\left|d_{g j}\right|$ if $d_{g}$ is an increased dependency and $S=\sum_{d_{g j}>0} d_{g j}$ if $d_{g}$ is a decreased dependency. Then, we define a weight function $W(M)$ that quantifies how well a subset $M=\left(m_{1}, \ldots, m_{k}\right)$ of features satisfies properties (i) and (ii). For the weight function $W(M)$, we generalize the weight function defined in [31] to measure the mutual exclusivity between mutations. Specifically, for a set $M$, let $\Gamma(M)$ be the subset of samples with alterations in $M, c_{j}(M)$ be the number of alterations in $M$ that occur in sample $j$, and $\rho_{j}$ be a penalty term for alterations in $M$ that co-occur in sample $j$. When searching for association to increased dependency, $\rho_{j}$ is equal to $-\left|d_{j}^{\prime}\right|$; when searching for association to decreased dependency, $\rho_{j}$ is equal to $\left|d_{j}^{\prime}\right|$. We define

$$
W(M)=\sum_{j \in \Gamma(M)} d_{j}^{\prime}-\left(c_{j}(M)-1\right) \rho_{j} .
$$


If the alterations in $M$ are mutually exclusive then $c_{j}(M)=1$ for all $j$ and thus $W(M)$ is the sum of differential dependency scores for all altered samples. If $c_{j}>1$, then sample $j$ has alterations in more than one feature in $M$, and thus we penalize the weight $W(M)$ for each additional alteration. Note that if the features that co-occur in a sample are GENE(I) and GENE(O) alterations, we do not penalize the weight. This is motivated by the two-hit hypothesis [101] which states that both alleles need to be mutated for gene inactivation. To see that the weight $W(M)$ is a straightforward generalization of the Dendrix weight introduced in [31] we consider the following reformulation, in which $\Gamma(m)$ denotes the set of samples with feature $m$.

$$
\begin{aligned}
W(M) & =\sum_{j \in \Gamma(M)} d_{j}^{\prime}-\left(c_{j}(M)-1\right) \rho_{j}=\sum_{j \in \Gamma(M)} d_{j}^{\prime}+\rho_{j}-c_{j}(M) \rho_{j} \\
& =\sum_{j \in \Gamma(M)}\left(d_{j}^{\prime}+\rho_{j}\right)-\sum_{m \in M} \sum_{j \in \Gamma(m)} \rho_{j}
\end{aligned}
$$

In the case where all samples have equal score, i.e., $d_{j}^{\prime}=1$, and $\rho_{j}=\left|d_{j}^{\prime}\right|=1$ for all $j$, the supervised Dendrix weight $W(M)$ simplifies to $W(M)=2|\Gamma(M)|-\sum_{m \in M}|\Gamma(m)|$, which is the original Dendrix weight defined in [31].

Following the nomenclature in machine learning, the problem considered in Dendrix [31] of finding a mutually exclusive set of alterations is an "unsupervised" feature selection problem, while the problem solved by SuperDendrix is a "supervised" feature selection problem where we aim to identify a set of mutually exclusive features that "explain" a phenotype.

Next, we aim to find a set $M^{*}$ with optimal weight $W\left(M^{*}\right)$, which we define as follows.

Problem 1 (Optimal Weight Exclusive Target Coverage Problem (OWXTC)). Given an alteration matrix $A$ and a differential dependency profile $d$, find a subset $M^{*}$ of rows satisfying

$$
M^{*}=\left\{\begin{array}{l}
\underset{M \subseteq \mathcal{E}}{\arg \min } W(M) \text { for increased dependencies, } \\
\underset{M \subseteq \mathcal{E}}{\arg \max } W(M) \text { for decreased dependencies, }
\end{array}\right.
$$

where $\mathcal{E}$ is all subsets of rows in $A$.

OWXTC is NP-hard because it generalizes the Maximum Weight Submatrix Problem which was shown to be NP-hard in [31] for the special case where $\mathbf{d}^{\prime}=(1,1, \ldots, 1)$ and $\rho=\mathbf{d}^{\prime}$. We also define the cardinality-constrained version $k$-OWTXC of OWXTC in which $\mathcal{E}$ is all subsets of size at most $k$.

We formulate the OWXTC as an integer linear program (ILP) as follows. First, we define binary variables $x_{i}$, for each row $1 \leq i \leq m$, and $y_{j}$, for each column $1 \leq j \leq n$, with the interpretation

$$
x_{i}=\left\{\begin{array}{ll}
1 & i \in M^{*} \\
0 & \text { otherwise }
\end{array} \quad \text { and } \quad y_{j}= \begin{cases}1 & a_{i j}=1 \text { for some } i \in M^{*} \\
0 & \text { otherwise. }\end{cases}\right.
$$

Then the OWXTC in the case of increased dependency is equivalent to the following ILP.

$$
\begin{array}{rrr}
\min & \sum_{d_{j}^{\prime}<0}\left(d_{j}^{\prime}+\rho_{j}\right) y_{j}-\sum_{i} \sum_{j \in \Gamma(i)} \rho_{j} x_{i} & \\
\text { subject to } & y_{j} \leq \sum_{i: a_{i j}=1} x_{i} & \text { for all } 1 \leq j \leq n \\
& y_{j} \geq x_{i} & \text { for all } i, j: a_{i j}=1 \text { and } w_{j}<0 \\
x_{i} \in\{0,1\} & \text { for all } 1 \leq i \leq m \\
y_{j} \in\{0,1\} & \text { for all } 1 \leq j \leq n
\end{array}
$$


For finding associations with decreased dependencies, we replace min by max in Equation (3). For the cardinality-restricted version, we add the inequality

$$
\sum_{i \in \mathcal{E}} x_{i} \leq k
$$

Note that the SuperDendrix weight and the ILP are similar, but not identical, to those presented in [26]. The differences are discussed in Supplementary Information.

\section{Evaluating statistical significance of associations}

The third module of SuperDendrix consists of two steps. First, since the optimal size $k=\left|M^{*}\right|$ of the feature set is unknown, we perform model selection using a conditional permutation test to evaluate the contribution of each alteration to the weight $W\left(M^{*}\right)$. For each feature $m$ in $M^{*}$, we compare the weight $W\left(M^{*}\right)$ to the distribution of the weight $W\left(\bar{M}^{*}\right)$, where $W\left(\bar{M}^{*}\right)$ is the weight obtained when mutations of the feature $m$ are permuted across samples. We compute the empirical $P$-value as $p_{m}=\operatorname{Pr}\left[W\left(\bar{M}^{*}\right) \leq\right.$ $\left.W\left(M^{*}\right)\right]$ (increased dependency) or $p_{m}=\operatorname{Pr}\left[W\left(\bar{M}^{*}\right) \geq W\left(M^{*}\right)\right]$ (decreased dependency) over 10,000 permutations and remove $m$ with the largest $P$-value only if $p_{m}>0.0001$. We repeat the above process until we obtain a feature set $\bar{M}$ which only contains features with $p_{m} \leq 0.0001$.

Next, we evaluate the statistical significance of the association between feature set $\bar{M}$ and differential dependency profile $\mathbf{d}$ by running SuperDendrix on random feature matrices $\hat{A}$ with fixed row and column sums (numbers of alterations per gene and sample, respectively) [102]. Note that we generate these random matrices using all alterations (i.e. including mutations not annotated in OncoKB), and then use the first module in SuperDendrix to select the OncoKB alteration features. We compare the weight $W(\bar{M})$ to the distribution of the weight $W(\hat{M})$, where $W(\hat{M})$ is the optimal weight computed from a random feature matrix $\hat{A}$. We compute the empirical $P$-value as $p=\operatorname{Pr}[W(\hat{M}) \leq W(\bar{M})$ (increased dependency) or $p=\operatorname{Pr}[W(\hat{M}) \geq W(\bar{M})$ (decreased dependency) over 10,000 random feature matrices.

After computing $P$-values of the feature sets for each differential dependency, we compute false discovery rate (FDR) using Benjamini-Hochberg procedure [103] for multiple hypothesis correction.

\section{Implementation and Software}

We implement SuperDendrix using Python 3. We use the R package, EMMIXskew [104], to fit $t$-distribution mixture models to dependency scores. We use the Python scikit-learn library [105] to fit Gaussian mixture models to dependency scores and to compute the $2 \mathrm{C}$ scores. We solve the ILP using the Gurobi software [106]. SuperDendrix software and our experiments are available at at https://github.com/ raphael-group/superdendrix.

\section{Bioinformatics and Data processing}

We downloaded the Avana [19Q1/2.19.2019] dataset from the DepMap data portal ${ }^{1}$ [39]. This dataset contains dependency scores - computed using the CERES algorithm - for 17,634 CRISPR-Cas9 gene knockouts across 558 cancer cell lines. We normalize each of 17,634 dependency profiles by converting CERES scores to z-scores as described in [39] before applying SuperDendrix. After running the first module of SuperDendrix, we obtain 492 differential dependencies that are better fit by a mixture of two $t$-distributions; 412 increased dependencies and 79 decreased dependencies.

We downloaded mutation data [19Q1/2.19.2019] for the same cell lines from the Cancer Cell Line Encyclopedia (CCLE) [43] using the same DepMap data portal. This dataset includes mutation data for 554

\footnotetext{
${ }^{1}$ https://depmap.org/portal/download/
} 
of the 558 cell lines in the CRISPR-Cas9 dataset. We applied SuperDendrix to 788,985 non-synonymous mutations. After running the first module of SuperDendrix we obtain an alteration matrix containing 566 alteration features (49 GENE(A), 229 GENE(I), and 228 GENE(O) alteration features) in 355 genes in 554 cell lines. Note that these alteration features do not overlap with the list of recently identified "passenger hotspot" mutations caused by preferential APOBEC activity in DNA stem loops [107]. To derive cancer-type features, we used the "primary_tissue" and "secondary_tissue" columns in the DepMap cell line metadata $^{2}$ and fixed annotation errors and merged rare cancer sub-types. Our annotation of cancer types is in "primary_tissue" column in our curated cell line data (Table S9]. We use this annotation to construct 31 binary cancer-type features representing the cancer types of DepMap cell lines where each feature has a value 1 for cell lines of that cancer type and 0 for other cell lines.

We run SuperDendrix using sets of at most 3 alterations and sets of at most 5 alterations and cancer types.

\section{Web browser for genetic dependency and mutation data}

We release a public, open-source web browser to view and explore SuperDendrix results. Users can choose which genetic dependency profile and which mutations they want to view, or can preload an association identified as significant by the SuperDendrix software. The browser displays a waterfall plot, indicating the dependency score and mutation status in each cell line. It also includes two bar plots on top of the waterfall plot that indicate tissue type and number of mutations per cell line. Users can interact with the plots by scrolling over bars in the waterfall plot. On mouse over, the browser displays tooltips listing information about the given cell line such as tissue type. Users can also select a range of cell lines in the bar plot at the top to zoom in. The plots provide an easy way to quickly assess whether the dependency scores in cell lines with user-specified alterations or cancer types are extreme relative to the other cell lines. The code for the SuperDendrix browser is open-source at https://github.com/lrgr/superdendrix-explorer, and the browser itself is publicly available at https://superdendrix-explorer.lrgr.io/

\section{Comparison to other methods}

As noted in the introduction, there are two other methods to find associations between mutually exclusive alterations and gene perturbation scores: REVEALER [25] and UNCOVER [26]. REVEALER uses a greedy method to find mutually exclusive alterations associated with continuous phenotype. As noted in [26], the greedy method is slow and not scalable to the large-scale Avana dataset containing thousands of dependency profiles, and therefore was not compared with SuperDendrix. UNCOVER was developed concurrently with our development of SuperDendrix, and also solves a combinatorial optimization problem. However, there are several key differences between SuperDendrix and UNCOVER.

1. UNCOVER is applied directly to dependency scores, while SuperDendrix first identifies and scores differential dependencies using a mixture model.

2. UNCOVER combines all mutations in a gene into a single gene-level alteration, while SuperDendrix creates different alteration features (GENE(A), GENE(I), or GENE(O)) according to OncoKB annotations.

3. UNCOVER uses a different objective function in the optimization with positive and negative scores having asymmetric contribution to the objective.

4. UNCOVER lacks a model selection step, and does not control for variability in the number of mutations across cell lines during its statistical test.

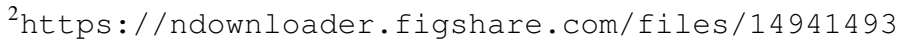


Further details of these differences are in Supplementary Information.

We compared SuperDendrix and UNCOVER on the same Avana dataset, and found that the methodological differences between SuperDendrix and UNCOVER led to large qualitative and quantitative differences in results. We ran UNCOVER using CERES scores of 1,730 $6 \sigma$ profiles and 9,987 alteration features and 31 cancer-type features (see Supplementary Information for data processing) to search for a set of 3 associated alterations features and 5 alteration or cancer-type features. UNCOVER reported 139 sets of alterations containing a total of 417 alterations with significant association (FDR $\leq 0.20$, Table S10), compared to 32 sets containing a total of 44 alterations for SuperDendrix. When the 31 cancer-type features were included, UNCOVER reported 722 associations compared to 135 for SuperDendrix (Table[S11).

There are multiple reasons for the larger number of associations predicted by UNCOVER. First, 71 of the 139 significant associations identified by UNCOVER are associations between dependencies that are not identified as differential dependencies by SuperDendrix. These include dependencies on ZNF592 and MAP3K2 whose dependency score distributions are unimodal (Fig. S9]. Second, UNCOVER does not include a model selection procedure, and thus always returns alteration sets of the requested size ( 3 and 5 in these experiments). Of the 24 differential dependencies where both UNCOVER and SuperDendrix reported associated alterations, UNCOVER's associated sets included 72 gene-level alterations $(24 \times 3)$, while SuperDendrix sets contain a total of 34 alterations (including GENE(A), GENE(I), and GENE(O) alterations). 29 of the gene-level alterations identified by UNCOVER overlap the 34 alterations identified by SuperDendrix in the corresponding profile. The remaining 43 gene-level alterations found by UNCOVER are not included in SuperDendrix results. Notably, 33 of these 43 alterations contribute less than $20 \%$ to the corresponding weight of the alteration set. Across UNCOVER's 139 total significant associations, $18 \%$ of the significant alterations $\left(\frac{74}{417}\right)$ contribute less than $20 \%$ to the set's weight. These alterations with small objective values are likely false positives. Finally, the permutation test used to evaluate statistical significance of UNCOVER's results does not control for variability in the number of mutations across cell lines. We found that the number of significant associations reported by UNCOVER in a cell line is significantly correlated with the number of alterations in the cell line (Pearson correlation: $R=0.85$ for alterations; and $R=0.83$ for alteration and cancer-type features) (Fig. S10, indicating that some of the associations reported by UNCOVER are likely false positives. In comparison, the correlation is much weaker in SuperDendrix results $(R=0.54$ for alterations only; and $R=0.27$ with cancer types included).

\section{Acknowledgements}

G.W.K. thanks the Fulbright Scholarship Program for support. This work is supported by US National Institutes of Health (NIH) grants R01HG007069 (B.J.R.) and U24CA211000 (B.J.R.).

\section{Declaration of Interests}

B.J.R. is a cofounder of, and consultant to, Medley

\section{References}

[1] Meyerson, M., Gabriel, S. \& Getz, G. Advances in understanding cancer genomes through secondgeneration sequencing. Nature Reviews Genetics 11, 685-696 (2010).

[2] Eifert, C. \& Powers, R. S. From cancer genomes to oncogenic drivers, tumour dependencies and therapeutic targets. Nature Reviews Cancer 12, 572-578 (2012). 
[3] Garraway, L. A. Genomics-driven oncology: framework for an emerging paradigm. Journal of Clinical Oncology 31, 1806-1814 (2013).

[4] Garraway, L. A. \& Lander, E. S. Lessons from the cancer genome. Cell 153, 17-37 (2013).

[5] Vogelstein, B. et al. Cancer genome landscapes. science 339, 1546-1558 (2013).

[6] Campbell, P. J. et al. Pan-cancer analysis of whole genomes. Nature 578, 82-93 (2020).

[7] Garraway, L. A. \& Sellers, W. R. Lineage dependency and lineage-survival oncogenes in human cancer. Nature Reviews Cancer 6, 593-602 (2006).

[8] Lovén, J. et al. Selective inhibition of tumor oncogenes by disruption of super-enhancers. Cell 153, 320-334 (2013).

[9] Bradner, J. E., Hnisz, D. \& Young, R. A. Transcriptional addiction in cancer. Cell 168, 629-643 (2017).

[10] Hofree, M. et al. Challenges in identifying cancer genes by analysis of exome sequencing data. Nature communications 7, 1-9 (2016).

[11] Luo, B. et al. Highly parallel identification of essential genes in cancer cells. Proceedings of the National Academy of Sciences 105, 20380-20385 (2008).

[12] Wang, T., Wei, J. J., Sabatini, D. M. \& Lander, E. S. Genetic screens in human cells using the CRISPR-Cas9 system. Science 343, 80-84 (2014).

[13] Shalem, O. et al. Genome-scale CRISPR-Cas9 knockout screening in human cells. Science 343, 84-87 (2014).

[14] McDonald III, E. R. et al. Project drive: a compendium of cancer dependencies and synthetic lethal relationships uncovered by large-scale, deep rnai screening. Cell 170, 577-592 (2017).

[15] Tsherniak, A. et al. Defining a Cancer Dependency Map. Cell 170, 564-570.e16 (2017).

[16] Behan, F. M. et al. Prioritization of cancer therapeutic targets using CRISPR-Cas9 screens. Nature 568, 511 (2019).

[17] Weinstein, I. B. \& Joe, A. Oncogene addiction. Cancer research 68, 3077-3080 (2008).

[18] Torti, D. \& Trusolino, L. Oncogene addiction as a foundational rationale for targeted anti-cancer therapy: promises and perils. EMBO molecular medicine 3, 623-636 (2011).

[19] Kaelin, W. G. The concept of synthetic lethality in the context of anticancer therapy. Nature reviews cancer 5, 689-698 (2005).

[20] Luo, J., Solimini, N. L. \& Elledge, S. J. Principles of cancer therapy: oncogene and non-oncogene addiction. Cell 136, 823-837 (2009).

[21] Boyle, E. A., Pritchard, J. K. \& Greenleaf, W. J. High-resolution mapping of cancer cell networks using co-functional interactions. Molecular systems biology 14 (2018).

[22] Kim, E. et al. A network of human functional gene interactions from knockout fitness screens in cancer cells. Life science alliance 2 (2019). 
[23] De Kegel, B. \& Ryan, C. J. Paralog buffering contributes to the variable essentiality of genes in cancer cell lines. PLoS genetics 15 (2019).

[24] Dempster, J. M. et al. Agreement between two large pan-cancer crispr-cas9 gene dependency data sets. Nature Communications 10, 1-14 (2019).

[25] Kim, J. W. et al. Characterizing genomic alterations in cancer by complementary functional associations. Nature Biotechnology 34, 539-546 (2016).

[26] Basso, R. S., Hochbaum, D. S. \& Vandin, F. Efficient algorithms to discover alterations with complementary functional association in cancer. PLoS computational biology 15, e1006802 (2019).

[27] Leiserson, M. D. et al. Pan-cancer network analysis identifies combinations of rare somatic mutations across pathways and protein complexes. Nature genetics 47, 106 (2015).

[28] Sanchez-Vega, F. et al. Oncogenic signaling pathways in the cancer genome atlas. Cell 173, 321-337 (2018).

[29] Yeang, C.-H., McCormick, F. \& Levine, A. Combinatorial patterns of somatic gene mutations in cancer. The FASEB journal 22, 2605-2622 (2008).

[30] Miller, C. A., Settle, S. H., Sulman, E. P., Aldape, K. D. \& Milosavljevic, A. Discovering functional modules by identifying recurrent and mutually exclusive mutational patterns in tumors. BMC medical genomics 4, 34 (2011).

[31] Vandin, F., Upfal, E. \& Raphael, B. J. De novo discovery of mutated driver pathways in cancer. Genome research 22, 375-385 (2012).

[32] Ciriello, G., Cerami, E., Sander, C. \& Schultz, N. Mutual exclusivity analysis identifies oncogenic network modules. Genome research 22, 398-406 (2012).

[33] Leiserson, M. D., Blokh, D., Sharan, R. \& Raphael, B. J. Simultaneous identification of multiple driver pathways in cancer. PLoS computational biology 9 (2013).

[34] Leiserson, M. D., Wu, H.-T., Vandin, F. \& Raphael, B. J. CoMEt: a statistical approach to identify combinations of mutually exclusive alterations in cancer. Genome Biology 16, 1061 (2015).

[35] Constantinescu, S., Szczurek, E., Mohammadi, P., Rahnenführer, J. \& Beerenwinkel, N. Timex: a waiting time model for mutually exclusive cancer alterations. Bioinformatics 32, 968-975 (2016).

[36] Chakravarty et al. OncoKB: A precision oncology knowledge base. JCO Precision Oncology 2017, $1-16$.

[37] Lawrence, M. S. et al. Mutational heterogeneity in cancer and the search for new cancer-associated genes. Nature 499, 214 (2013).

[38] Bailey, M. H. et al. Comprehensive characterization of cancer driver genes and mutations. Cell 173, 371-385 (2018).

[39] Meyers et al. Computational correction of copy number effect improves specificity of CRISPRCas9 essentiality screens in cancer cells. Nature Genetics 49, 1779.

[40] Forbes, S. A. et al. Cosmic: mining complete cancer genomes in the catalogue of somatic mutations in cancer. Nucleic acids research 39, D945-D950 (2010). 
[41] Gene Ontology Consortium. The gene ontology resource: 20 years and still GOing strong. Nucleic acids research 47, D330-D338 (2018).

[42] Mi, H., Muruganujan, A., Ebert, D., Huang, X. \& Thomas, P. D. PANTHER version 14: more genomes, a new PANTHER GO-slim and improvements in enrichment analysis tools. Nucleic acids research 47, D419-D426 (2018).

[43] Barretina, J. et al. The cancer cell line encyclopedia enables predictive modelling of anticancer drug sensitivity. Nature 483, 603-607 (2012).

[44] Canning, P., Sorrell, F. J. \& Bullock, A. N. Structural basis of Keap1 interactions with Nrf2. Free Radical Biology and Medicine 88, 101-107 (2015).

[45] Padmanabhan, B. et al. Structural basis for defects of Keap1 activity provoked by its point mutations in lung cancer. Molecular cell 21, 689-700 (2006).

[46] UniProt Consortium. Uniprot: a worldwide hub of protein knowledge. Nucleic acids research 47, D506-D515 (2018).

[47] Arkenau, H., Kefford, R. \& Long, G. Targeting braf for patients with melanoma. British journal of cancer 104, 392-398 (2011).

[48] Helming, K. C. et al. Arid1b is a specific vulnerability in arid1a-mutant cancers. Nature medicine 20, 251 (2014).

[49] Cloer, E. W., Goldfarb, D., Schrank, T. P., Weissman, B. E. \& Major, M. B. NRF2 activation in cancer: from DNA to protein. Cancer research 79, 889-898 (2019).

[50] Leinonen, H. M., Kansanen, E., Pölönen, P., Heinäniemi, M. \& Levonen, A.-L. Dysregulation of the Keap1-Nrf2 pathway in cancer. Biochemical Society Transactions 43, 645-649 (2015).

[51] Kensler, T. W. \& Wakabayashi, N. Nrf2: friend or foe for chemoprevention? Carcinogenesis 31, 90-99 (2010).

[52] Kansanen, E., Kuosmanen, S. M., Leinonen, H. \& Levonen, A.-L. The Keap1-Nrf2 pathway: mechanisms of activation and dysregulation in cancer. Redox biology 1, 45-49 (2013).

[53] Kitamura, H. \& Motohashi, H. Nrf2 addiction in cancer cells. Cancer science 109, 900-911 (2018).

[54] Nevins, J. R. The Rb/E2F pathway and cancer. Human molecular genetics 10, 699-703 (2001).

[55] Eskandarpour, M., Huang, F., Reeves, K. A., Clark, E. \& Hansson, J. Oncogenic NRAS has multiple effects on the malignant phenotype of human melanoma cells cultured in vitro. International journal of cancer 124, 16-26 (2009).

[56] Waters, A. M. \& Der, C. J. KRAS: the critical driver and therapeutic target for pancreatic cancer. Cold Spring Harbor perspectives in medicine 8, a031435 (2018).

[57] Poulikakos, P. I. \& Rosen, N. Mutant BRAF melanomasdependence and resistance. Cancer cell 19, $11-15$ (2011).

[58] Leicht, D. T. et al. Raf kinases: function, regulation and role in human cancer. Biochimica et Biophysica Acta (BBA)-Molecular Cell Research 1773, 1196-1212 (2007). 
[59] Kaplan, F. M. et al. SHOC2 and CRAF mediate ERK1/2 reactivation in mutant NRAS-mediated resistance to RAF inhibitor. Journal of Biological Chemistry jbc-M112 (2012).

[60] Flaherty, K. T. et al. Improved survival with mek inhibition in braf-mutated melanoma. New England Journal of Medicine 367, 107-114 (2012).

[61] Qin, J., Xin, H. \& Nickoloff, B. J. Specifically targeting erk1 or erk2 kills melanoma cells. Journal of translational medicine 10, 15 (2012).

[62] Lister, J. A. et al. A conditional zebrafish MITF mutation reveals MITF levels are critical for melanoma promotion vs. regression in vivo. Journal of Investigative Dermatology 134, 133-140 (2014).

[63] Cagnol, S. \& Rivard, N. Oncogenic KRAS and BRAF activation of the MEK/ERK signaling pathway promotes expression of dual-specificity phosphatase 4 (DUSP4/MKP2) resulting in nuclear ERK1/2 inhibition. Oncogene 32, 564 (2013).

[64] Chen, H.-F., Chuang, H.-C. \& Tan, T.-H. Regulation of dual-specificity phosphatase (DUSP) ubiquitination and protein stability. International journal of molecular sciences 20, 2668 (2019).

[65] Gröschl, B. et al. Expression of the MAP kinase phosphatase DUSP4 is associated with microsatellite instability in colorectal cancer (CRC) and causes increased cell proliferation. International journal of cancer 132, 1537-1546 (2013).

[66] Teutschbein, J. et al. Gene expression analysis after receptor tyrosine kinase activation reveals new potential melanoma proteins. BMC cancer 10, 386 (2010).

[67] Amin, A. D., Rajan, S. S., Groysman, M. J., Pongtornpipat, P. \& Schatz, J. H. Oncogene overdose: Too much of a bad thing for oncogene-addicted cancer cells. Biomarkers in cancer 7, BIC-S29326 (2015).

[68] Chen, Y.-N. P. et al. Allosteric inhibition of SHP2 phosphatase inhibits cancers driven by receptor tyrosine kinases. Nature 535, 148 (2016).

[69] Coste, I. et al. Dual function of MyD88 in RAS signaling and inflammation, leading to mouse and human cell transformation. The Journal of clinical investigation 120, 3663-3667 (2010).

[70] Yu, X. et al. Myd88 1265p mutation in lymphoid malignancies. Cancer research 78, 2457-2462 (2018).

[71] Hoffman, G. R. et al. Functional epigenetics approach identifies brm/smarca2 as a critical synthetic lethal target in brg1-deficient cancers. Proceedings of the National Academy of Sciences 111, 31283133 (2014).

[72] Benedetti, L., Cereda, M., Monteverde, L., Desai, N. \& Ciccarelli, F. D. Synthetic lethal interaction between the tumour suppressor stag2 and its paralog stag1. Oncotarget 8, 37619 (2017).

[73] Lambert, S. A. et al. The human transcription factors. Cell 172, 650-665 (2018).

[74] Durbin et al. Selective gene dependencies in MYCN-amplified neuroblastoma include the core transcriptional regulatory circuitry. Nature Genetics 50, 1240-1246.

[75] Aspland, S. E., Bendall, H. H. \& Murre, C. The role of e2a-pbx1 in leukemogenesis. Oncogene 20, 5708-5717 (2001). 
[76] Mullighan, C. G. Molecular genetics of b-precursor acute lymphoblastic leukemia. The Journal of clinical investigation 122, 3407-3415 (2012).

[77] Rohde, M. et al. Relevance of ID3-TCF3-CCND3 pathway mutations in pediatric aggressive Bcell lymphoma treated according to the non-Hodgkin lymphoma Berlin-Frankfurt-Münster protocols. Haematologica 102, 1091-1098 (2017).

[78] Wöhner, M. et al. Molecular functions of the transcription factors E2A and E2-2 in controlling germinal center B cell and plasma cell development. Journal of Experimental Medicine 213, 12011221 (2016).

[79] Gloury, R. et al. Dynamic changes in Id3 and E-protein activity orchestrate germinal center and plasma cell development. Journal of Experimental Medicine 213, 1095-1111 (2016).

[80] Lin, C. Y. et al. Mapping of the multiple myeloma transcriptional core regulatory circuitry reveals tcf3 as a novel dependency and an oncogenic collaborator of myc. Blood 130, 64-64 (2017).

[81] Pon, J. R. et al. Mef2b mutations in non-hodgkin lymphoma dysregulate cell migration by decreasing mef2b target gene activation. Nature communications 6, 1-15 (2015).

[82] Kwak, Y. et al. C-MET overexpression and amplification in gliomas. International journal of clinical and experimental pathology 8, 14932 (2015).

[83] Visvader, J. E., Mao, X., Fujiwara, Y., Hahm, K. \& Orkin, S. H. The LIM-domain binding protein Ldb1 and its partner LMO2 act as negative regulators of erythroid differentiation. Proceedings of the National Academy of Sciences 94, 13707-13712 (1997).

[84] Valge-Archer, V., Forster, A. \& Rabbitts, T. The LMO1 and LDB1 proteins interact in human t cell acute leukaemia with the chromosomal translocation t $(11 ; 14)(\mathrm{p} 15 ; \mathrm{q} 11)$. Oncogene 17, 3199 (1998).

[85] Zhao, Q., Tran, H., Dimitrov, D. S. \& Cheung, N.-K. V. A dual-specific anti-IGF-1/IGF-2 human monoclonal antibody alone and in combination with temsirolimus for therapy of neuroblastoma. International journal of cancer 137, 2243-2252 (2015).

[86] Perini, G. F., Ribeiro, G. N., Neto, J. V. P., Campos, L. T. \& Hamerschlak, N. BCL-2 as therapeutic target for hematological malignancies. Journal of hematology \& oncology 11, 65 (2018).

[87] Lamers, F. et al. Targeted bcl 2 inhibition effectively inhibits neuroblastoma tumour growth. European Journal of Cancer 48, 3093-3103 (2012).

[88] Metz, H. E. \& Houghton, A. M. Insulin receptor substrate regulation of phosphoinositide 3-kinase. Clinical Cancer Research 17, 206-211 (2011).

[89] Li, R., Pourpak, A. \& Morris, S. W. Inhibition of the insulin-like growth factor-1 receptor (igf1r) tyrosine kinase as a novel cancer therapy approach. Journal of medicinal chemistry 52, 4981-5004 (2009).

[90] Wainberg, M. et al. A genome-wide almanac of co-essential modules assigns function to uncharacterized genes. bioRxiv 827071 (2019).

[91] Dang, C. V., Reddy, E. P., Shokat, K. M. \& Soucek, L. Drugging the 'undruggable' cancer targets. Nature Reviews Cancer 17, 502 (2017). 
[92] Leiserson, M. D., Wu, H.-T., Vandin, F. \& Raphael, B. J. Comet: a statistical approach to identify combinations of mutually exclusive alterations in cancer. Genome biology 16, 160 (2015).

[93] Geisinger, J. M. \& Stearns, T. Crispr/cas9 treatment causes extended tp53-dependent cell cycle arrest in human cells. bioRxiv 604538 (2019).

[94] Fortin, J.-P. et al. Multiple-gene targeting and mismatch tolerance can confound analysis of genomewide pooled crispr screens. Genome biology 20, 21 (2019).

[95] Leiserson, M. D. M., Blokh, D., Sharan, R. \& Raphael, B. J. Simultaneous identification of multiple driver pathways in cancer. PLoS Computational Biology 9, e1003054 (2013).

[96] Yang, W. et al. Genomics of drug sensitivity in cancer (gdsc): a resource for therapeutic biomarker discovery in cancer cells. Nucleic acids research 41, D955-D961 (2012).

[97] Masica, D. L. \& Karchin, R. Collections of simultaneously altered genes as biomarkers of cancer cell drug response. Cancer research 73, 1699-1708 (2013).

[98] Knijnenburg, T. A. et al. Logic models to predict continuous outputs based on binary inputs with an application to personalized cancer therapy. Nature Scientific Reports 1-14 (2016).

[99] Peel, D. \& McLachlan, G. J. Robust mixture modelling using the t distribution. Statistics and computing 10, 339-348 (2000).

[100] Schwarz, G. et al. Estimating the dimension of a model. The annals of statistics 6, 461-464 (1978).

[101] Knudson, A. G. Mutation and cancer: statistical study of retinoblastoma. Proceedings of the National Academy of Sciences 68, 820-823 (1971).

[102] Strona, G., Nappo, D., Boccacci, F., Fattorini, S. \& San-Miguel-Ayanz, J. A fast and unbiased procedure to randomize ecological binary matrices with fixed row and column totals. Nature communications 5, 4114 (2014).

[103] Benjamini, Y. \& Hochberg, Y. Controlling the false discovery rate: a practical and powerful approach to multiple testing. Journal of the Royal statistical society: series B (Methodological) 57, 289-300 (1995).

[104] Wang, K., Ng, A., McLachlan, G. \& Lee, M. S. Package emmixskew (2018).

[105] Pedregosa, F. et al. Scikit-learn: Machine learning in Python. Journal of Machine Learning Research 12, 2825-2830 (2011).

[106] Gurobi Optimization, LLC. Gurobi optimizer reference manual (2018). URL http://www. gurobi.com.

[107] Buisson, R. et al. Passenger hotspot mutations in cancer driven by APOBEC3A and mesoscale genomic features. Science 364, eaaw2872 (2019).

[108] Staudt, L. M. Oncogenic activation of NF- $\kappa$ B. Cold Spring Harbor perspectives in biology 2 , a000109 (2010).

[109] Xia, Y., Shen, S. \& Verma, I. M. NF- $\kappa$ B, an active player in human cancers. Cancer immunology research 2, 823-830 (2014). 
[110] Roy, P., Sarkar, U. \& Basak, S. The NF- $\kappa$ B activating pathways in multiple myeloma. Biomedicines 6, 59 (2018).

[111] Hankey, W., Frankel, W. L. \& Groden, J. Functions of the APC tumor suppressor protein dependent and independent of canonical WNT signaling: implications for therapeutic targeting. Cancer and Metastasis Reviews 37, 159-172 (2018).

[112] van der Meer, D. et al. Cell model passportsa hub for clinical, genetic and functional datasets of preclinical cancer models. Nucleic acids research 47, D923-D929 (2019).

[113] Westermark, U. K., Wilhelm, M., Frenzel, A. \& Henriksson, M. A. The MYCN oncogene and differentiation in neuroblastoma. In Seminars in cancer biology, vol. 21, 256-266 (Elsevier, 2011).

[114] Huang, M. \& Weiss, W. A. Neuroblastoma and MYCN. Cold Spring Harbor perspectives in medicine 3, a014415 (2013).

[115] Mundo, L. et al. Molecular switch from MYC to MYCN expression in MYC protein negative Burkitt lymphoma cases. Blood cancer journal 9, 1-10 (2019).

[116] Roussigne, M., Cayrol, C., Clouaire, T., Amalric, F. \& Girard, J.-P. THAP1 is a nuclear proapoptotic factor that links prostate-apoptosis-response-4 (Par-4) to PML nuclear bodies. Oncogene 22, 2432 (2003).

[117] Cayrol, C. et al. The THAP-zinc finger protein THAP1 regulates endothelial cell proliferation through modulation of pRB/E2F cell-cycle target genes. Blood 109, 584-594 (2007).

[118] Krieghoff-Henning, E. \& Hofmann, T. G. Role of nuclear bodies in apoptosis signalling. Biochimica et Biophysica Acta (BBA)-Molecular Cell Research 1783, 2185-2194 (2008).

[119] Giacomelli, A. O. et al. Mutational processes shape the landscape of tp53 mutations in human cancer. Nature genetics 50, 1381 (2018). 


\section{Supplementary Figures}

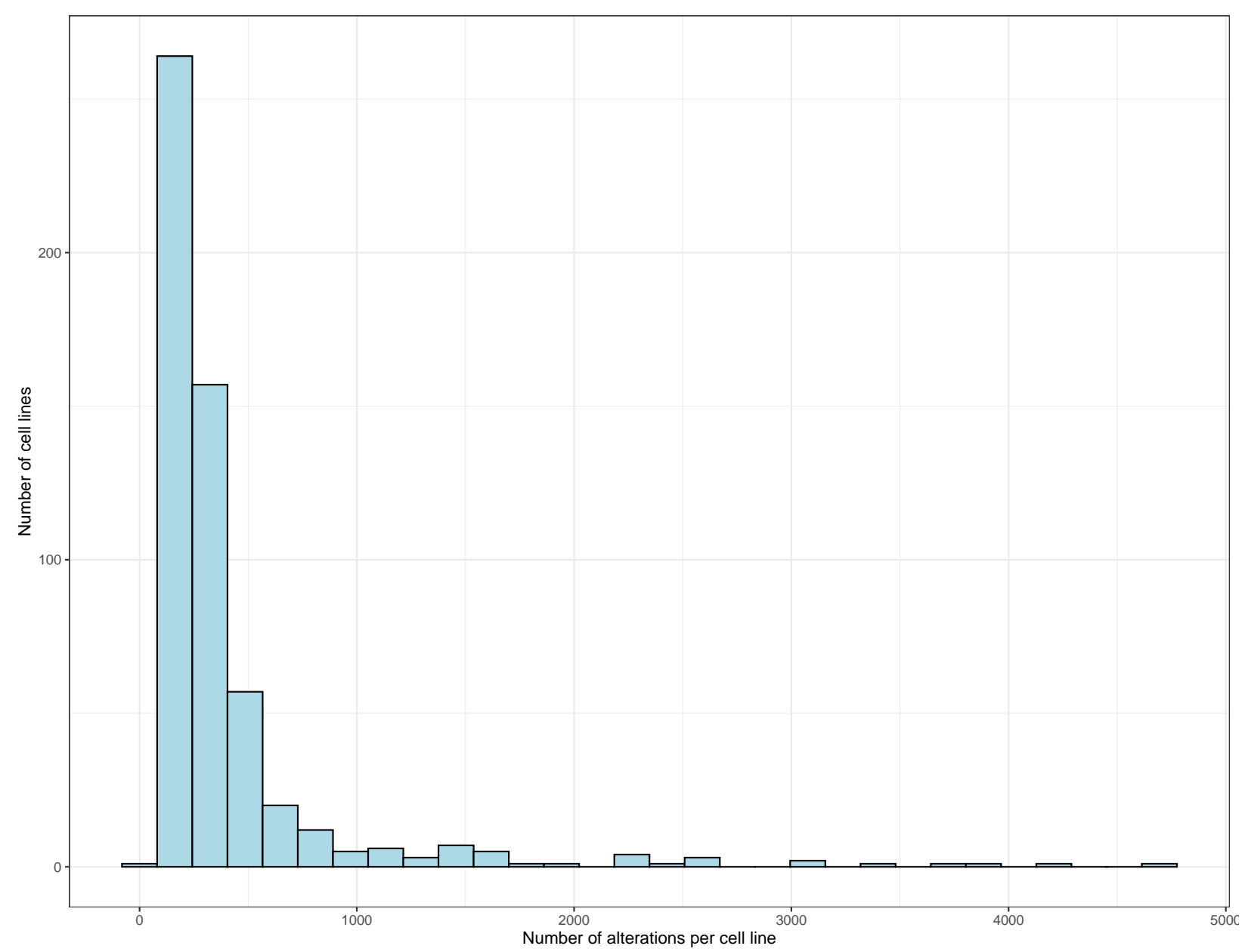

Figure S1: Number of genomic alterations in cell lines in DepMap. Distribution of all non-synonymous mutations in the cell lines from DepMap. Mean $=410.01$ and standard deviation $=533.43$.

Figure S2: Results of fitting a mixture of two t-distributions to the 17,634 dependency profiles. Attached as a supplement file. 


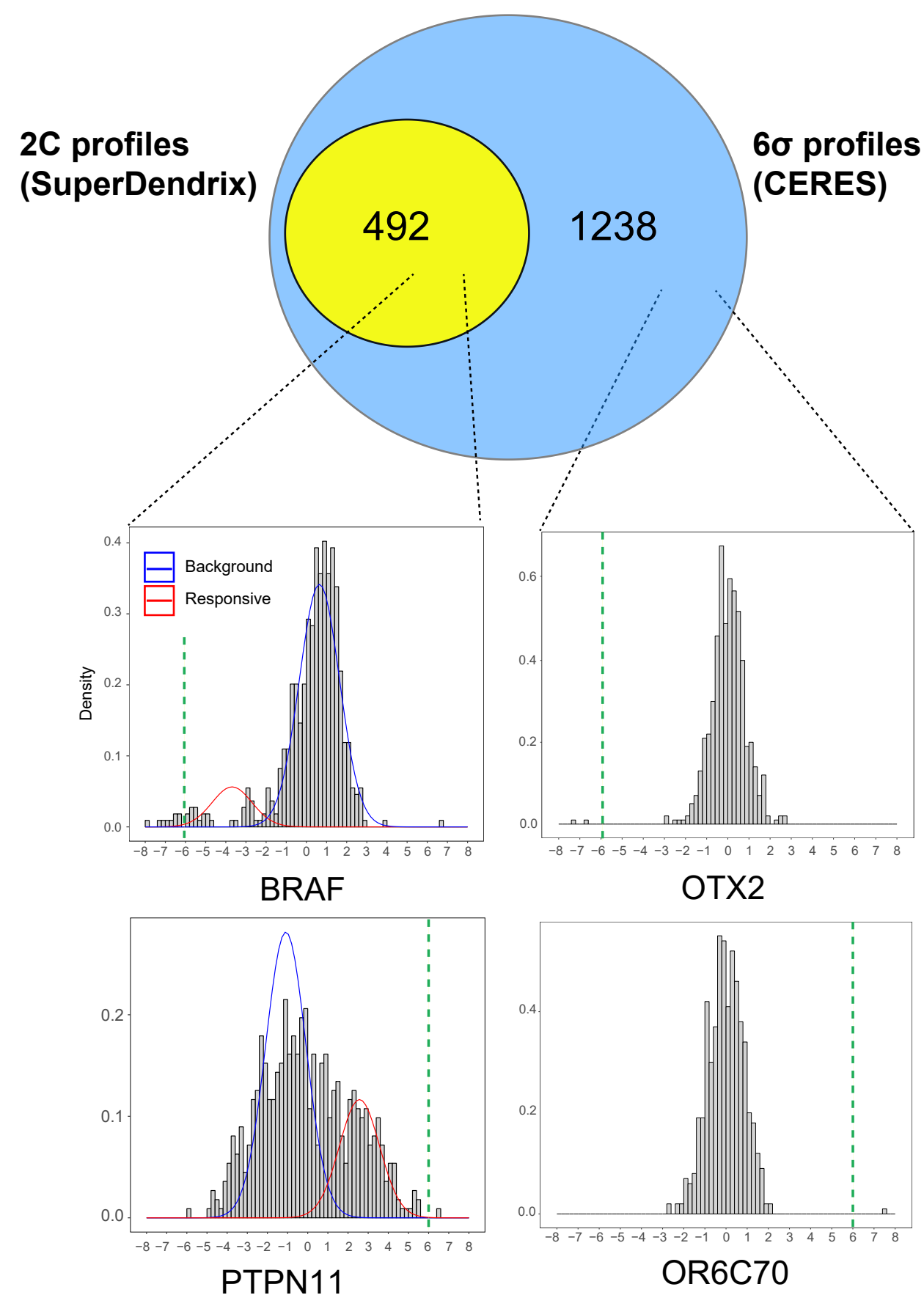

Figure S3: Comparison of differential dependencies found by SuperDendrix and CERES. (Top) Overlap between differential dependencies identified using the two component mixture model in SuperDendrix and the $6 \sigma$ threshold for CERES z-scores as described in [15]. (Bottom) CERES z-score distributions for example profiles from each section of Venn diagram. The top row are profiles with increased dependency upon gene knockout, while the bottom row are profiles with decreased dependency. Red and blue curves indicate distributions in two component mixture, and green dashed lines represent $6 \sigma$ thresholds for CERES Z-scores. 


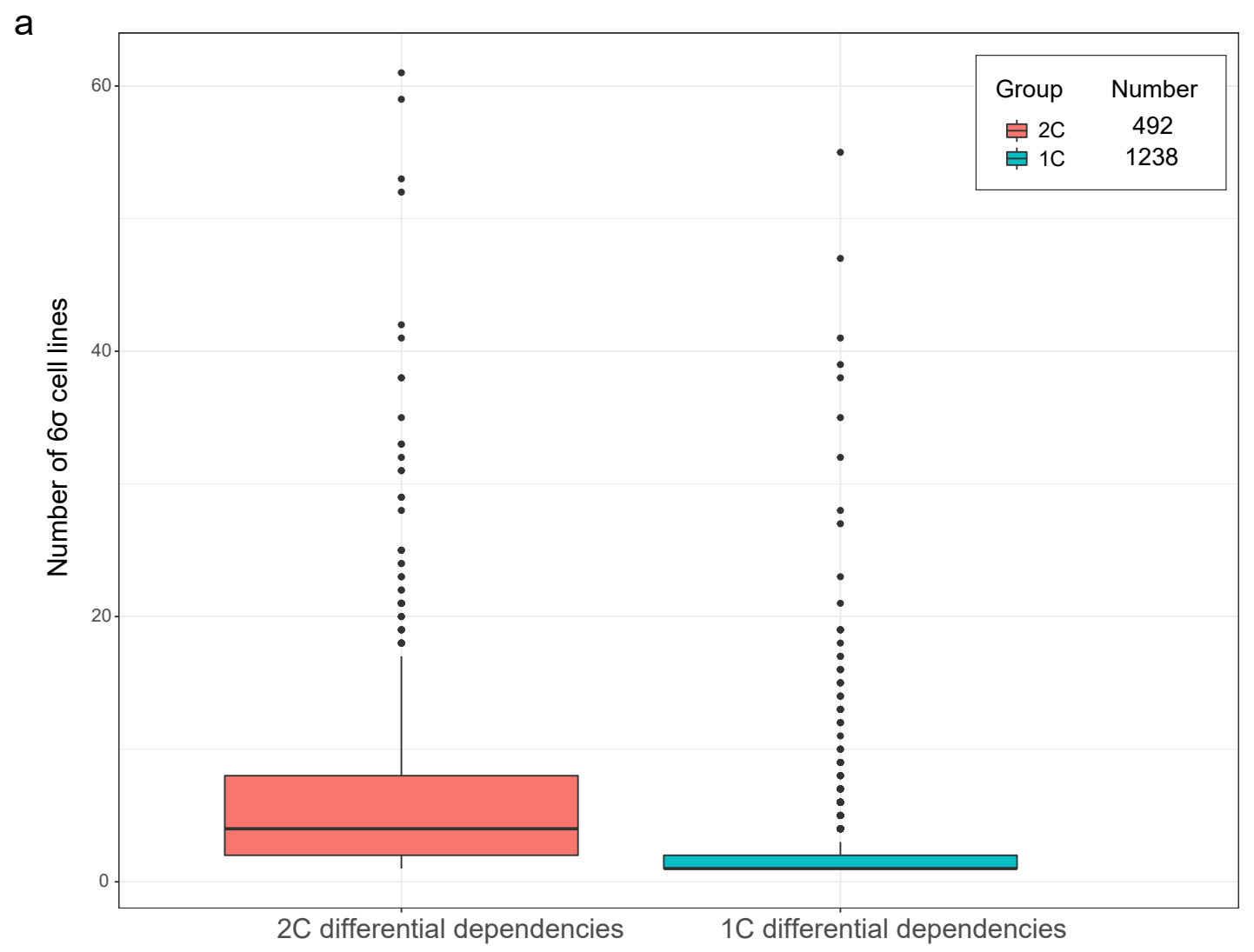

b

TSC2

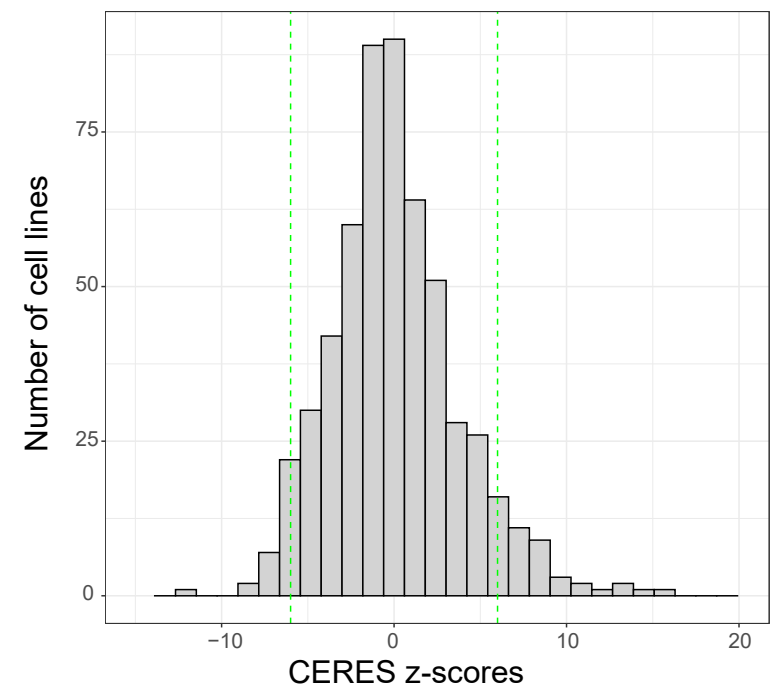

NF2

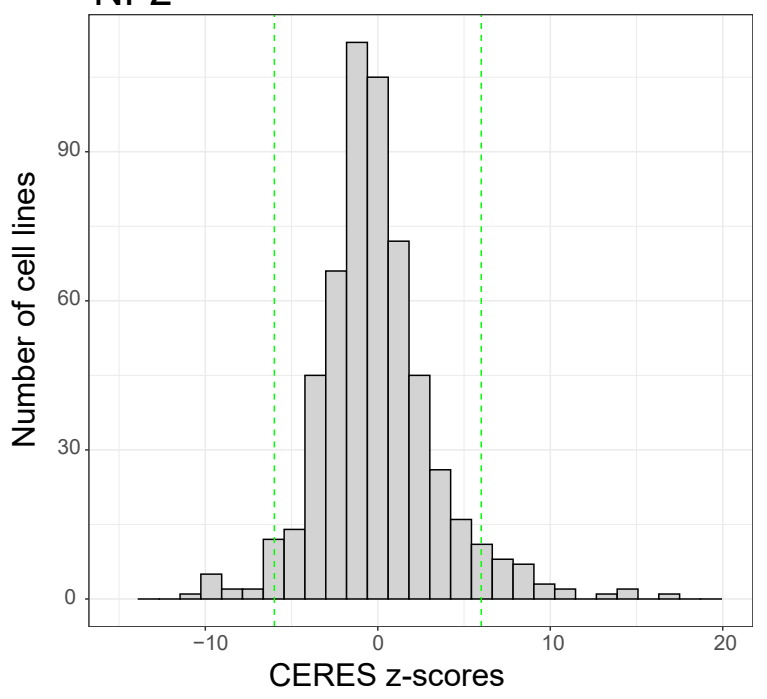

Figure S4: Number of $6 \sigma$ outlier cell lines in $2 \mathrm{C}$ and $1 \mathrm{C}$ profiles. (a) Box plots of number of cell lines with CERES scores $\geq 6 \sigma$ from the mean for $2 \mathrm{C}$ profiles and $1 \mathrm{C}$ profiles. $2 \mathrm{C}$ profiles contain higher number of $6 \sigma$ cell lines than 1C profiles on average (Welch t-test, $P<0.001$ ). (b) CERES z-score distributions of TSC2 and NF2, the 1C profiles containing the highest number of $6 \sigma$ outlier cell lines (55 and 47). The outlier distributions have wide standard deviations: 3.73 and 3.42. The green dashed lines represent $6 \sigma$ thresholds for CERES z-scores. 
bioRxiv preprint doi: https://doi.org/10.1101/2020.07.13.184697; this version posted July 14,2020 . The copyright holder for this preprint (which was not certified by peer review) is the author/funder, who has granted bioRxiv a license to display the preprint in perpetuity. It is made available under aCC-BY-NC-ND 4.0 International license.
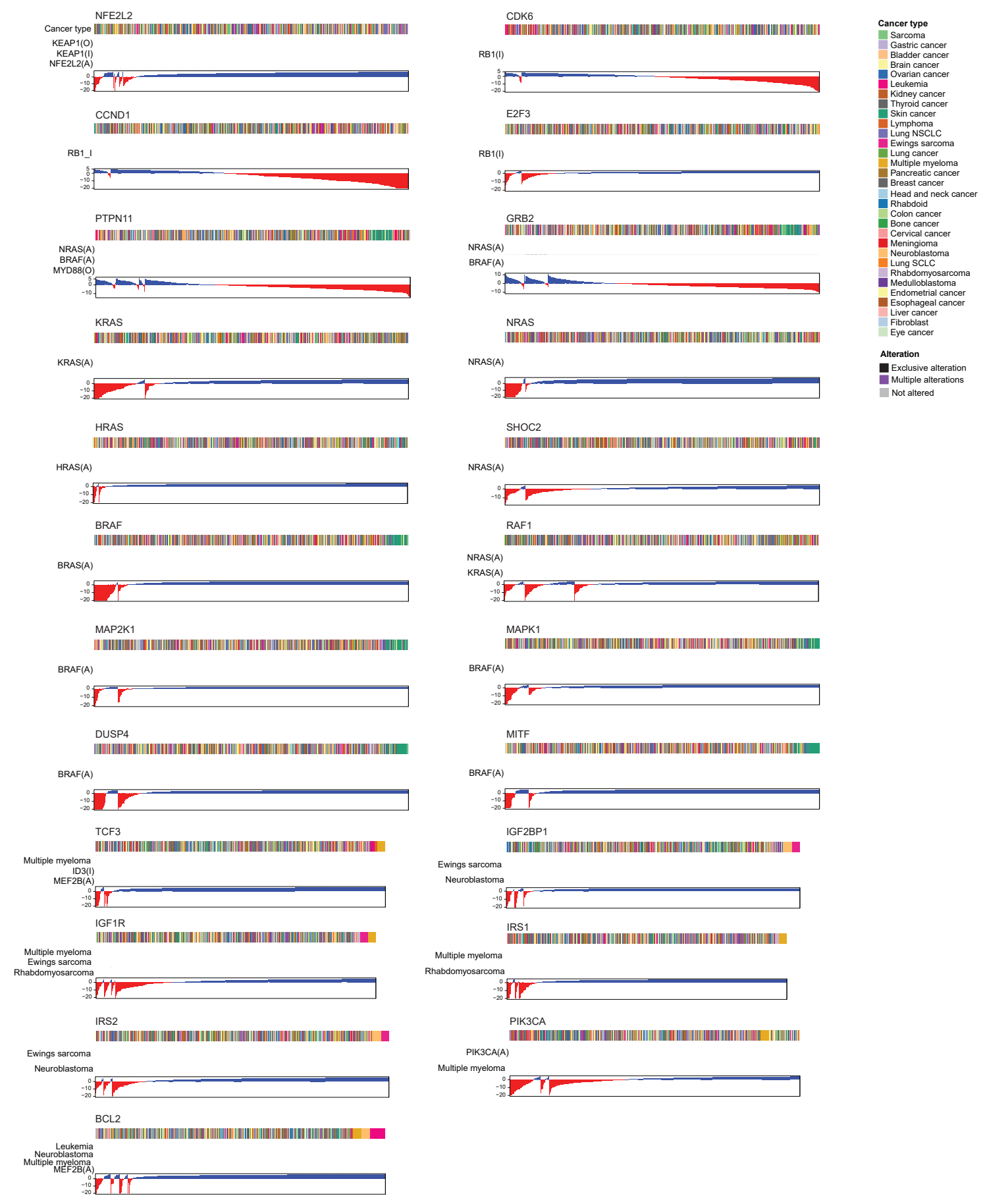

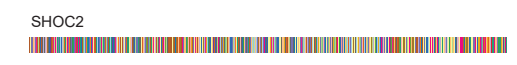
NRAS(A)

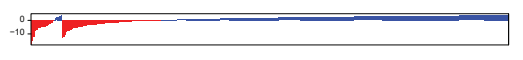

RAF1

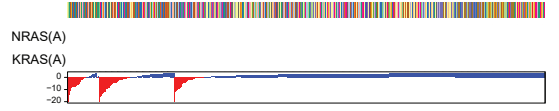

MAPK

MAPK1 BRAF(A)

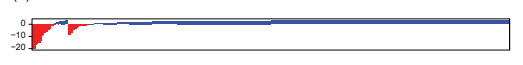

MITF

| BRAF(A)
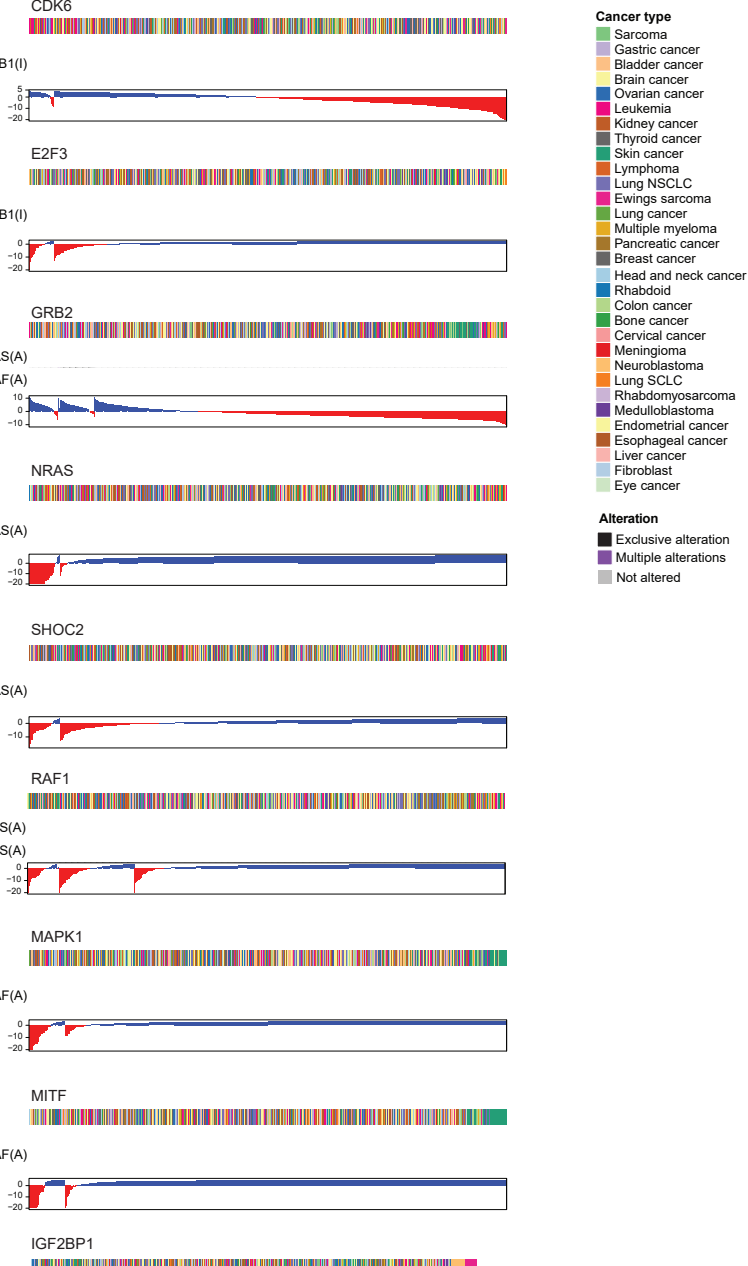
Ewings sarcoma

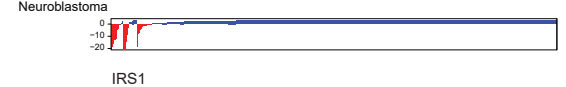

Multiple myeloma

IRS1

Rhabdomyosarcoma

垈 PIK3CA(A)

PIK3CA Multiple myeloma

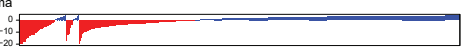

Figure S5: Alteration matrices and profiles for significant associations discovered by SuperDendrix. Blue boxes are cell lines that contain the corresponding alteration. Red boxes are cell lines that contain multiple alterations in each association. 


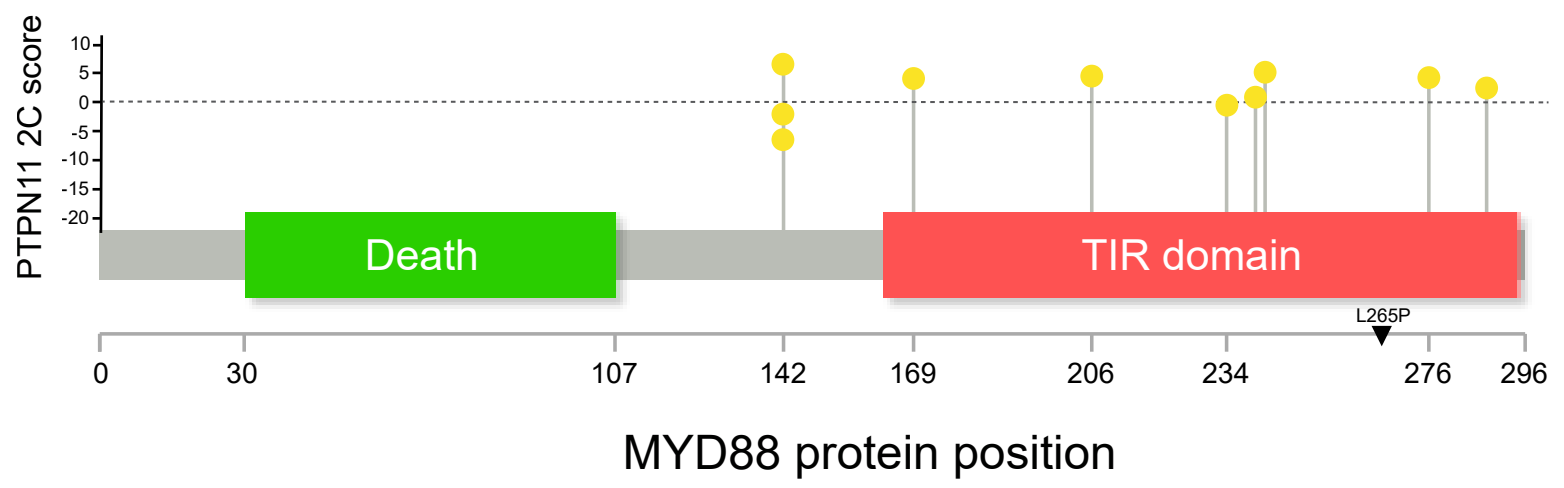

Figure S6: Locations of mutations in MYD88 protein that are annotated as Other. MYD88(0) mutations are associated with decreased dependency on PTPN11. 7 of the 10 MYD88(0) mutations occur in TIR domain $(P=0.11$; binomial test). CERES scores for $P T P N 11$ are higher (decreased dependency) on average in the 7 cell lines with MYD88(0) mutations in TIR domain than the 3 cell lines with MYD88(0) mutations outside of TIR $(P=0.13 ;$ t-test $)$. 


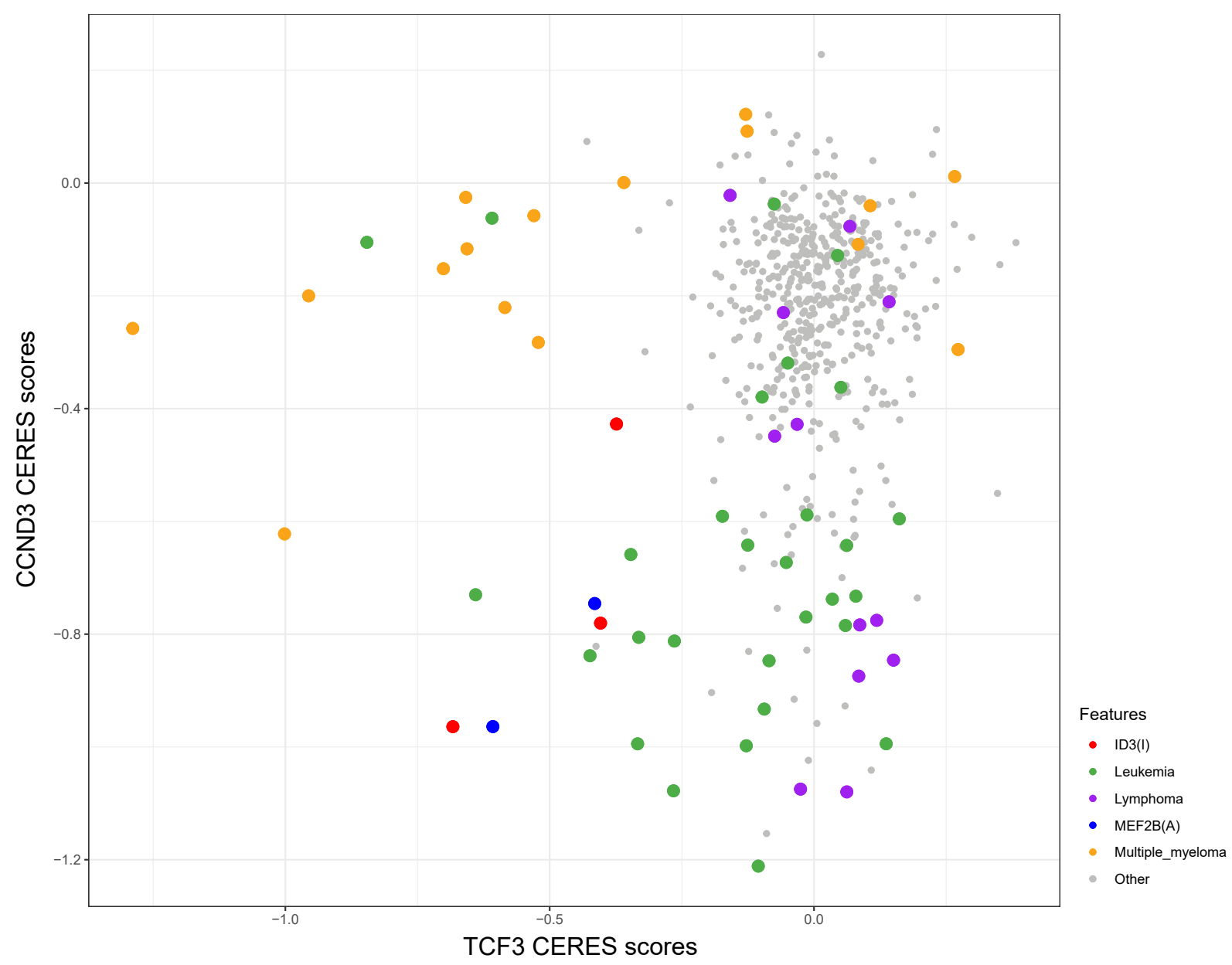

Figure S7: Increased dependencies on TCF3 and CCND3 in blood cancers. CERES dependency scores for TCF3 (x-axis) and CCND3 (y-axis) across cell lines with multiple myeloma (orange), leukemia (green), and lymphoma cell (purple) cell lines showing a mutually exclusive pattern with increased dependency on either TCF3 and CCND3. In contrast, the subset of B-cell lymphomas containing ID3(I) (red) or MEF2B(A) (blue) mutations show increased dependencies on both TCF3 and CCND3. 


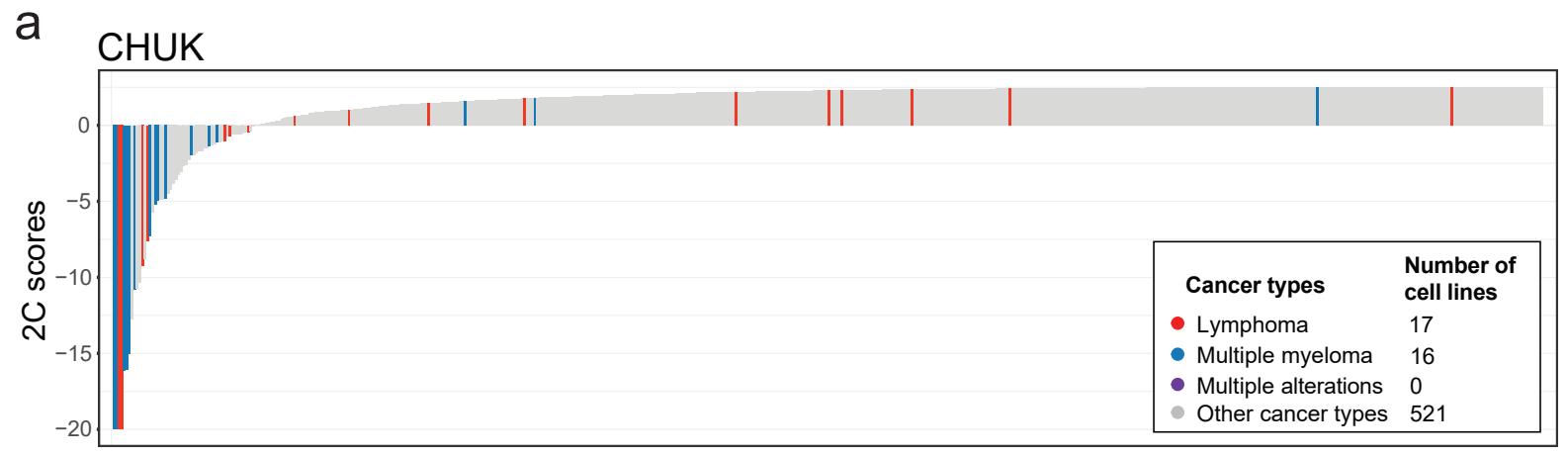

IKK complex
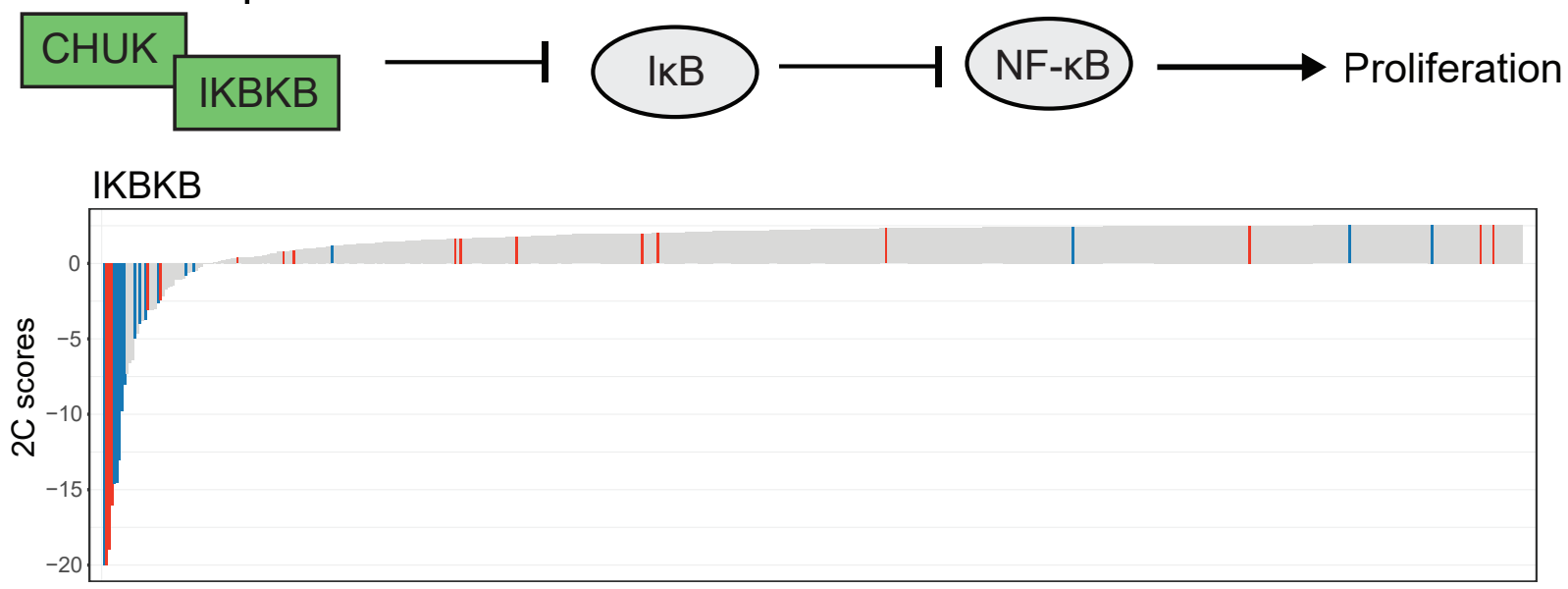

Cell lines

b

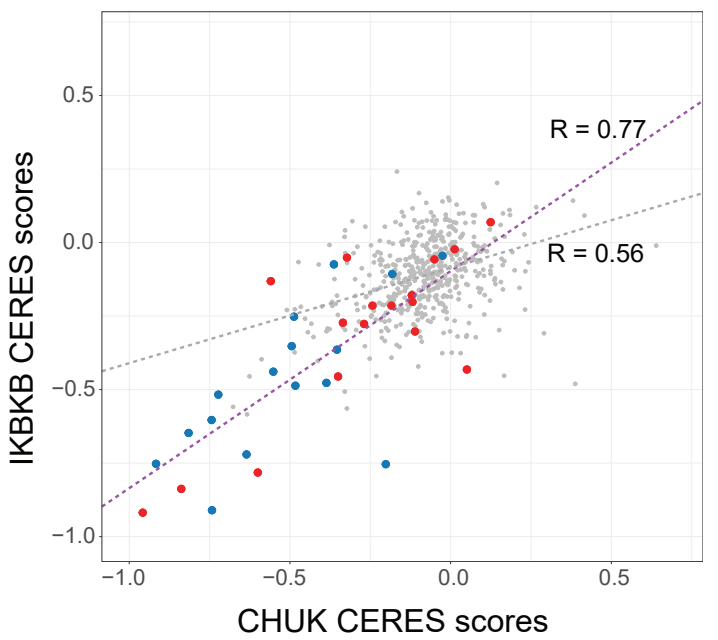

Figure S8: Cancer-type specific differential dependencies in NF- $\kappa$ B pathway. (a) Lymphoma and multiple myeloma are associated with increased dependency on $C H U K$ and $I K B K B$, the $\alpha$ and $\beta$ subunits of IKK complex, that activate downstream NF- $\kappa$ B pathway (Same format as Fig. 2b). This is consistent with previous reports of NF- $\kappa \mathrm{B}$ deregulation in blood cancers [108, 109, 110]. (b) CERES scores of cancer types that are differentially dependent in both $C H U K$ and $I K B K B$ profiles show positive correlation $(R=0.77$, $P<0.001)$. 

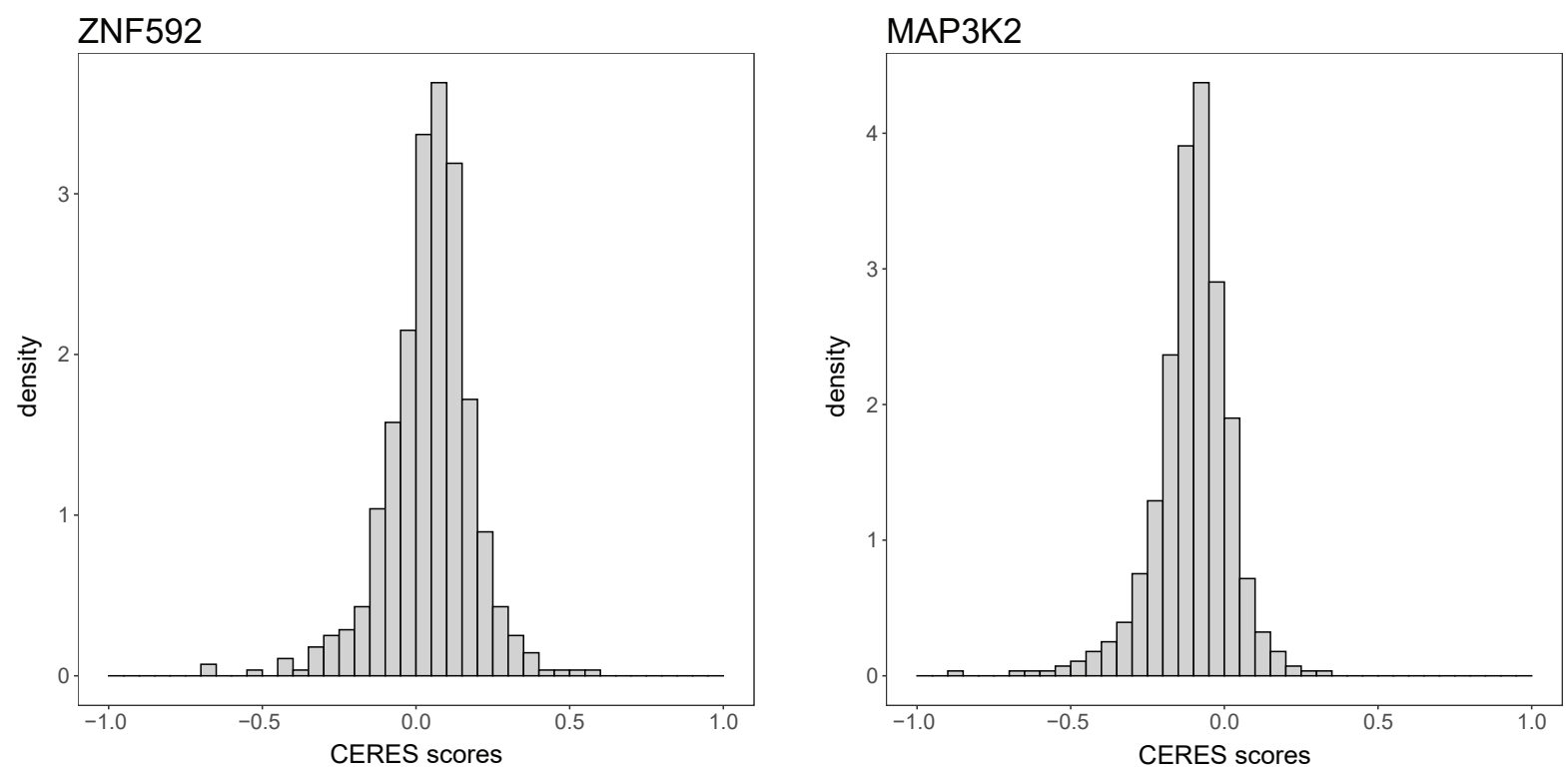

Figure S9: UNCOVER finds associations between alterations and dependencies with unimodal score distribution. CERES score distributions of ZNF592 and MAP3K2. Each of these distributions is unimodal and better fit by a single t-distribution than by a mixture of $2 \mathrm{t}$-distributions.

a

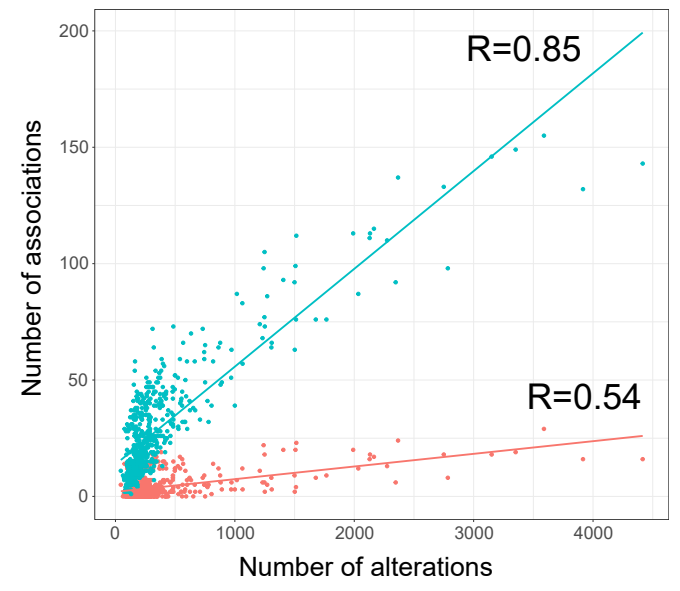

b

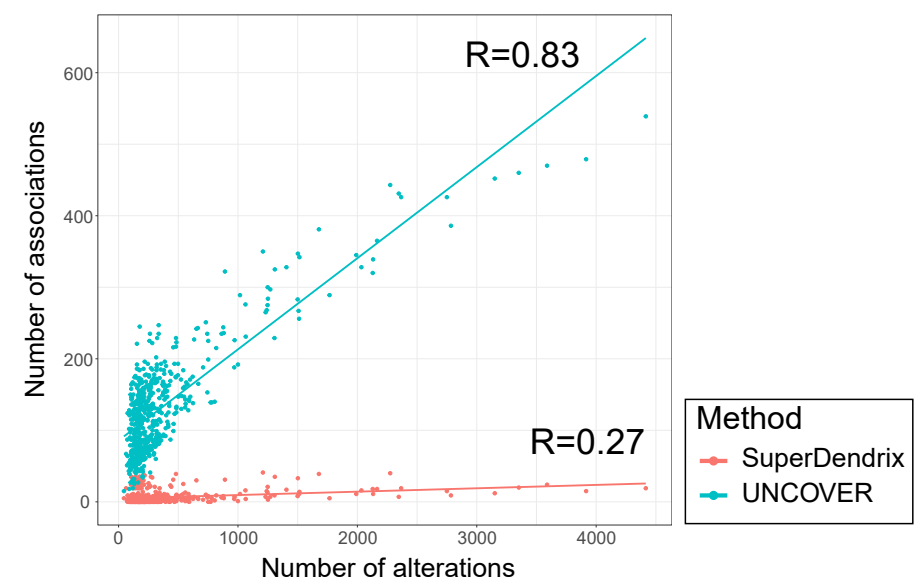

Figure S10: Correlation between total number of alterations and alterations from significant associations in each cell line.(a)-(b) Correlation was computed by comparing the total number of alterations (prior to OncoKB filtering) against the number of significant features in each cell line. (a) Correlation is lower in SuperDendrix for alteration results (SuperDendrix: $R=0.54$, UNCOVER: $R=0.85$ ). (b) Correlation is lower in SuperDendrix also for cancer type results (SuperDendrix: $R=0.27$, UNCOVER: $R=0.83$ ). 

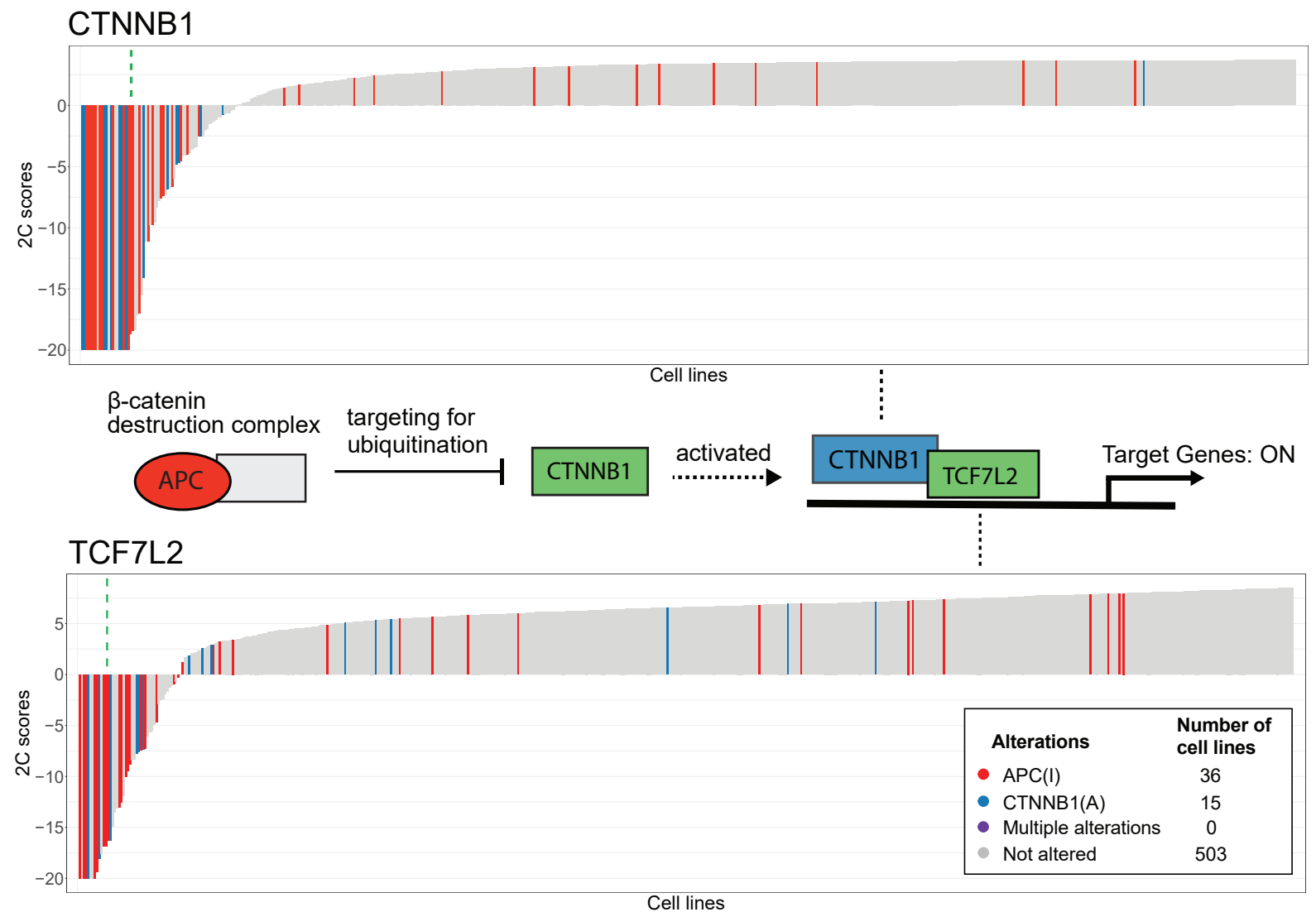

Figure S11: SuperDendrix identifies dependencies in the Wnt pathway. Cell lines with $A P C$ inactivating and CTNNB1 activating alterations are associated with increased dependency on CTNNB1 and TCF7L2 (Same format as Fig. 2b). These associations are consistent with the known interactions on Wnt signaling pathway. 


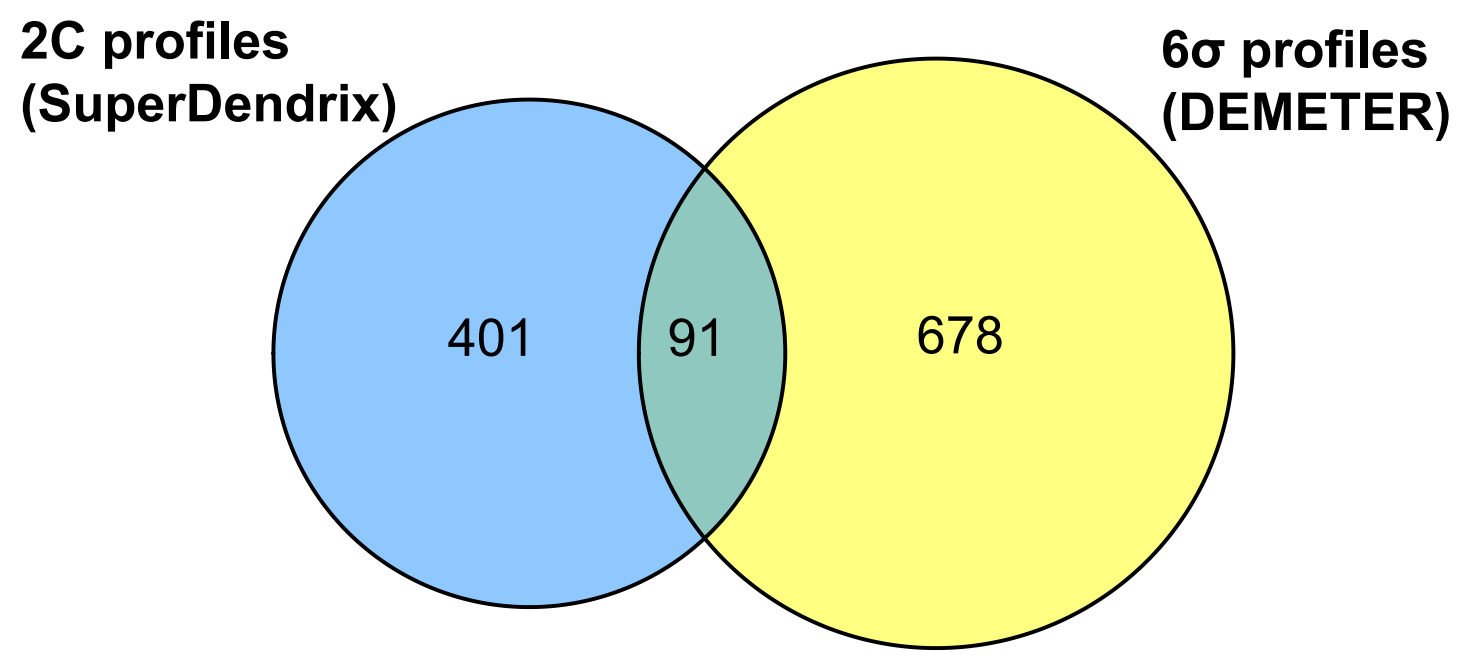

Figure S12: Comparison of differential dependencies found by SuperDendrix and DEMETER. Overlap between 492 differential dependencies identified by SuperDendrix from CRISPR dataset vs. 769 differential dependencies identified using $6 \sigma$ cutoff in RNAi dataset from [15].

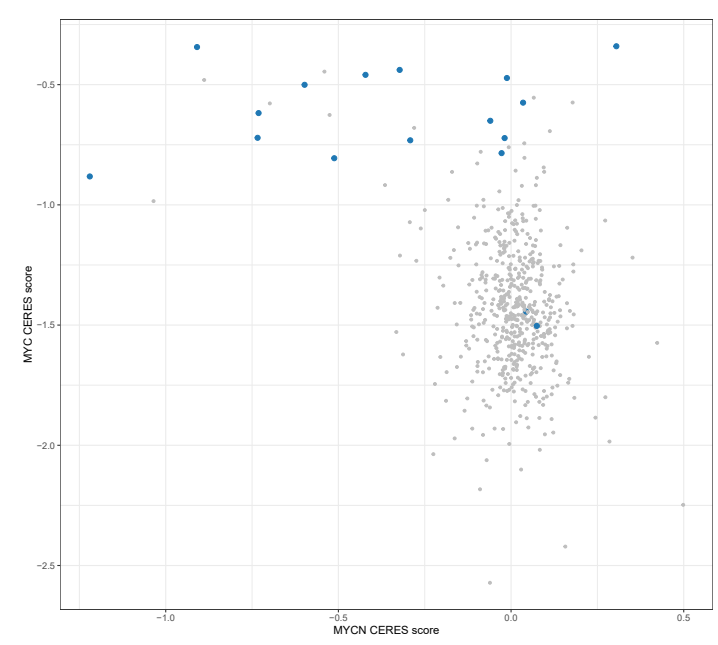

(a) Expression

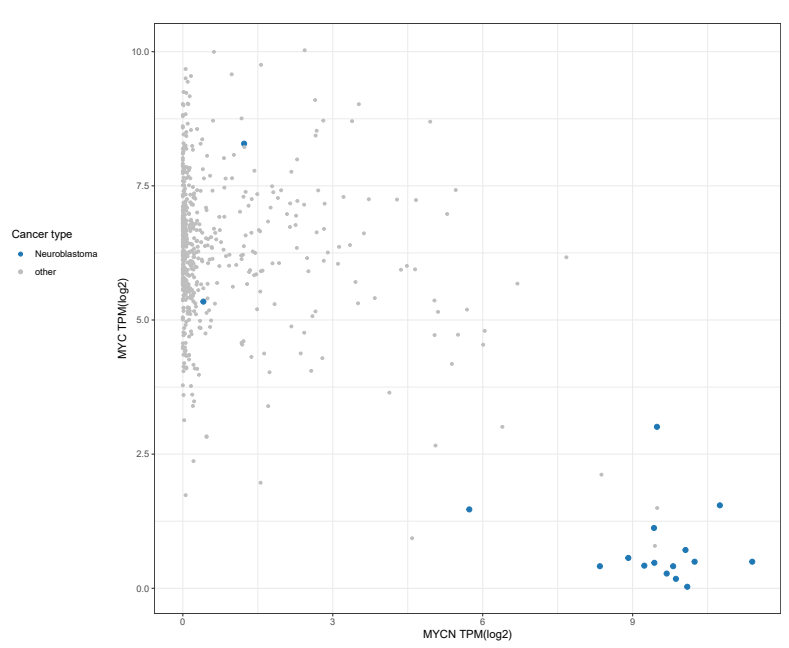

(b) CERES scores

Figure S13: Dependencies on $M Y C$ and $M Y C N$ in neuroblastoma. (a) $M Y C$ is associated with decreased dependency in neuroblastoma. In contrast, $M Y C N$ is associated with increased dependency in neuroblastoma. (b) Consistent with these associations, $M Y C$ expression is high across cancer types except for neuroblastoma while $M Y C N$ expression is low except for the same cancer type. 


\section{Supplementary Information}

\section{Associations in Wnt pathway}

SuperDendrix finds two associations in the Wnt pathway, including an association between $\operatorname{APC}(\mathrm{I})$ and CTNNB1(A) alterations and increased dependency in both CTNNB1 and TCF7L2 (Fig. S11). Alterations in APC(I) and CTNNB1(A) are mutually exclusive with no cell line containing both. Moreover, these dependencies are consistent with previously reported interactions in the Wnt pathway: when APC and correspondingly the $\beta$-catenin destruction complex are inactivated, CTNNB1 binds TCF7L2 in the nucleus and activates transcription of target genes [111].

\section{Comparison to other perturbation screen results}

We compare the differential dependencies and alteration sets associated with these dependencies identified with our methods to the results of RNAi screening from Tsherniak et al. [15] and CRISPR-Cas9 screening from Behan et al. [16].

Tsherniak et al. [15] identified $6 \sigma$ genes and associated genomic markers of these differential dependencies using RNAi screens of 501 cancer cell lines as part of the DepMap project. This analysis is distinct from ours in terms of the perturbation assay (RNAi instead of CRISPR) and score (DEMETER [15] instead of CERES), and in that Tsherniak et al. consider copy number aberrations and gene expression data - in addition to mutations - as potential genomic markers. There are 313 cell lines shared between the RNAi and CRISPR datasets.

We first compare in terms of differential dependencies. Tsherniak et al. [15] analyzed 6,305 profiles that pass quality control and identified $7696 \sigma$ genes. 91 of these $6 \sigma$ profiles are also among the $4922 \mathrm{C}$ differential dependencies (Fig. S12). Despite the small number of overlaps, the two sets of differential dependency profiles represent similar classes of proteins. In particular, both sets are significantly enriched for GO molecular functions such as DNA binding and protein kinase activity. They also contain similar proportion of CGC genes (Tsherniak et al. $6 \sigma: 12.2 \% P<0.01,2 \mathrm{C}: 18.7 \% P<0.01$ ). Genes that are unique to each set also capture similar GO molecular functions including nucleotide binding, protein binding, and $\mathrm{G}$ protein-coupled receptor activity and are both significantly enriched for CGC genes $(P<$ $0.01)$.

We next compare our results with Tsherniak et al. [15] in terms of biomarkers for differential dependency. Tsherniak et al. used a random forest-based approach to identify genomic features that are predictive of differential dependency, which they referred to as "marker dependency pairs" (MDPs). Using mutations, copy number aberrations, and gene expression, Tsherniak et al. [15] found MDPs for 426 of the $7696 \sigma$ profiles in the RNAi data. However, only 10 of these correspond to mutation driven biomarkers. In contrast, SuperDendrix found significantly associated mutation sets in 32 of 492 2C differential dependencies in the CRISPR data. 5 biomarker associations (mutation driven) are identified by both methods. These include well-known associations such as oncogene addictions of $B R A F, N R A S$, and KRAS. Interestingly, associations identified only by SuperDendrix include those with strong evidence, such as RAF1 dependency on KRAS or NRAS mutations, STAG1 dependency on STAG2 mutations, and NFE2L2 dependency on KEAP1 mutations.

As part of DepMap project, Behan et al. [16] independently conducted genome-wide CRISPR-Cas9 loss-of-function screens in 324 cancer cell lines. From a total of 18,009 knockout genes, they identified 628 priority targets based on combination of gene knockout effect across cell lines and their associations to biomarkers (single nucleotide variants, copy number variations, and microsatellite instability status). 147 of the priority targets are also among the $4922 \mathrm{C}$ profiles from SuperDendrix. The two sets of genes are significantly enriched for GO molecular functions such as DNA binding, protein binding, and transcription 
regulator activity. They also contain similar proportion of CGC genes (priority targets: $15.9 \% P<0.01,2 \mathrm{C}$ : $18.7 \% P<0.01)$.

Behan et al. analyzed associations of gene knockout effects with genomic biomarkers within each cancer type using ANOVA. Associations that occur across multiple cancer types were aggregated and re-tested using a $t$-test across all cell lines. We compare our results to their associations to SNVs considering all cell lines since we do not test for cancer-type-specific biomarker associations. Behan et al. identified a total of 41 significant biomarker associations $(F D R \leq 0.20)$ in 30 of the 628 priority target genes. However, only 10 associations for 8 genes are with SNV biomarkers. 3 of these (KRAS-KRASmut, PIK3CA-PIK3CAmut, GRB2-KRASmut) are also identified by SuperDendrix.

Overall, we are able to explain 6.5\% (32) of 2C differential dependencies (492) with alterations using SuperDendrix, 9 of which contain more than one alteration. In contrast, Tsherniak et al. [15] and Behan et al. [16] can each explain only $1.3 \%\left(\frac{10}{769}\right)$ and $1.6 \%\left(\frac{10}{628}\right)$ of their differential dependencies with mutation features. These findings indicate that our model, by searching for a set of approximately mutually exclusive alterations, has higher sensitivity for identifying associations between gene dependency biomarkers.

\section{Validation on the Sanger CRISPR-Cas9 screen data}

We used the dataset from genome-wide CRISPR-Cas9 screens conducted as part of the Cancer Dependency Map at Wellcome Sanger Institute [16] to validate the associations identified by SuperDendrix from the Avana dataset of the Cancer Dependency Map at the Broad Institute.

First, we downloaded the Score dataset containing dependency scores computed from results of CRISPR screens across 324 cancer cell lines from the Project Score data portal ${ }^{3}$ and a list of mutations for the same cell lines from Cell Model Passports data portal ${ }^{4}$ [112]. We used quantile normalized log fold-changes as dependency scores and processed the mutation data using SuperDendrix OncoKB feature selection. We restricted our validation to 312 cell lines that contain at least one OncoKB mutation feature. For each association identified by SuperDendrix in the Avana dataset, we compared the dependency scores of cell lines containing at least one of the features with dependency scores of the cell lines without any feature. We found that 22 of the 32 associations between differential dependencies and mutations identified by SuperDendrix and 72 of the 117 associations between differential dependencies and mutations or cancer-type features identified by SuperDendrix are statistically significant in the Score dataset $(P \leq 0.05$; Wilcoxon rank sum test). We find that many of the associations identified by SuperDendrix that did not validate in the Score dataset are in cancer types that were poorly represented in the Score dataset (Table S5).

\section{Decreased dependency on transcription factors}

Three of the 41 transcription factors with cancer-type specific differential dependencies, MYC, THAPl, TP53, show decreased dependency in specific cancer types. For example, we find decreased dependency on $M Y C$ in neuroblastoma. Interestingly, neuroblastoma has increased dependency on MYCN - a well-known oncogene for neuroblastoma [113, 114] - another member of the same family of MYC genes (Fig. S13(a)). $M Y C N$ is highly expressed in a subset of tissue including forebrain and hindbrain whereas $M Y C$ expression is ubiquitous [114]. Previous studies have suggested that MYCN and MYC may be redundant, and that MYCN can compensate MYC activity [114, 115]. In fact, we observe that MYC expression is high across most cancer types in the dataset, with neuroblastoma a prominent exception; at the same time, MYCN expression is low across most cancer types except for neuroblastoma (Fig. S13(b)). Within neuroblastoma, expression of MYC and MYCN is approximately mutually exclusive, with most neuroblastoma cell lines

$\sqrt[3]{\text { https://score.depmap.sanger.ac.uk/downloads }}$

4 https://cellmodelpassports.sanger.ac.uk/downloads 
expressing MYCN but not MYC. Based on these, we hypothesize that our result of the decreased dependency on MYC in neuroblastoma is because of its low expression in neuroblastoma, a result of redundant function and compensatory activity by MYCN. This result is consistent with the expected relationship between gene expression and dependency: cell lines in which a gene is not expressed will not be affected by knockout of that gene. As another example, we find decreased dependency on THAP1 in leukemia and lymphoma. THAP1 is known as a pro-apoptotic factor involved in regulating endothelial cell proliferation and linking PAWR to promyelocytic leukemia (PML) nuclear bodies (NB) [116, 117]. Interaction of PAWR and PML has been reported to trigger apoptosis [118]. Furthermore, PML is a tumor suppressor primarily expressed in blood vessels and a negative regulator of cell survival pathways [116, 118]. These reports on lineage-specificity and function of THAP1 and PML suggest that knocking out THAP1 which leads to loss of PML function resulted in decreased dependency or even prolonged cell survival in leukemia and lymphoma. We also find decreased dependency on TP53 in rhapdoid, kidney cancer, and skin cancer. A possible explanation for decreased dependency on TP53 is its wild-type function as a tumor suppressor. A previous study reports that knocking out TP53 in cells with functional wild-type TP53 where p53 acts as a tumor suppressor will induce growth advantage in those cells [119]. In our results, we noticed that many of the rhapdoid, kidney cancer, and skin cancer cell lines contain wild-type TP53. We thus tested for association between decreased dependency on TP53 and TP53(WT) as an additional feature using SuperDendrix. In fact, SuperDendrix identified a significant association between them $(P \leq 0.001)$, confirming that this is a decreased dependency conferred by inhibiting tumor suppressor activity of p53 in TP53 wild-type cell lines as suggested by [119].

\section{UNCOVER model}

Although the underlying integer linear programming formulations differ, the optimization model of UNCOVER is similar to the SuperDendrix weight. Using the same notation from the SuperDendrix weight, the UNCOVER objective function is the following:

$$
w(M)=\sum_{j \in \Gamma(M)} d_{j}-\left(c_{j}(M)-1\right) p_{j}
$$

This function consists of two terms, $d_{j}$ and $p_{j}$, that represent association to phenotype and penalty for co-occurring alterations. While UNCOVER uses the same linear term as SuperDendrix for biomarkerphenotype association, it uses a penalty term that has different values depending on the sign of the phenotype score. Specifically, if the phenotype score $d_{j}$ in cell line $j$ is positive, then UNCOVER sets the penalty $p_{j}$ to the average of the positive phenotype scores; alternatively if the phenotype score $d_{j}$ in cell line $j$ is negative then UNCOVER sets the penalty $p_{j}$ to be the absolute value of the score.

\section{Data preprocessing for UNCOVER}

We use the same CERES data and alteration data ${ }^{5}$. For consistency, we process the phenotype scores and alteration features as described in UNCOVER. For phenotype scores, we normalize CERES scores in each knockout profile by subtracting the mean of the profile for each score and dividing the result by standard deviation of the profile. Since mixture model is a procedure specific to SuperDendrix pipeline, we run UNCOVER on 1,730 $6 \sigma$ profiles. For alterations, we combine nonsense, frameshift, and missense mutations of each gene as a single alteration feature. A gene alteration in a cell line exists if at least one of nonsense, frameshift, or missense mutation is found in that cell line. This results in 9,987 alteration features occurring across 554 cell lines.

5 https://depmap.org/portal/download/api/download/external?file_name=ccle\% 2Fdepmap-mutation-calls-9ala.9\%2Fdepmap_19Q1_mutation_calls_v2.csv 


\section{Supplementary Tables}

All of the supplementary tables are attached in a supplementary information file.

Table S1: Information on $6 \sigma$ differential dependencies from CERES scores.

Table S2: Results of fitting t-mixture models to CERES dependency profiles.

Table S3: Enrichment for GO molecular functions in 2C profiles.

\begin{tabular}{|l|l|l|l|l|}
\hline Profile & Alterations & W(M) & FDR & Validation P-value \\
\hline NRAS & NRAS(A) & -0.898 & 0 & 0.000 \\
\hline BRAF & BRAF(A) & -0.885 & 0 & 0.000 \\
\hline KRAS & KRAS(A) & -0.81 & 0 & 0.000 \\
\hline MAPK1 & BRAF(A) & -0.662 & 0 & 0.000 \\
\hline ARID1B & ARID1A(I) & -0.546 & 0 & 0.000 \\
\hline SOX10 & BRAF(A) & -0.486 & 0 & 0.953 \\
\hline DUSP4 & BRAF(A) & -0.479 & 0 & 0.653 \\
\hline RAF1 & NRAS(A),KRAS(A) & -0.468 & 0 & 0.000 \\
\hline PTPN11 & BRAF(A),NRAS(A),MYD88(O) & 0.439 & 0 & 0.000 \\
\hline GRB2 & BRAF(A),NRAS(A) & 0.419 & 0 & 0.000 \\
\hline STAG1 & STAG2(I) & -0.417 & 0 & 0.008 \\
\hline PIK3CA & PIK3CA(A) & -0.396 & 0 & 0.000 \\
\hline DOCK5 & KRAS(A) & -0.379 & 0 & 0.000 \\
\hline SHOC2 & NRAS(A) & -0.29 & 0 & 0.000 \\
\hline CCND1 & RB1(I) & 0.098 & 0 & 0.000 \\
\hline PEA15 & BRAF(A) & -0.622 & 0.003 & 0.001 \\
\hline CTNNB1 & APC(I),CTNNB1(A) & -0.613 & 0.003 & 0.000 \\
\hline MITF & BRAF(A) & -0.452 & 0.003 & 0.623 \\
\hline STX4 & TP53(I),TP53(O) & -0.369 & 0.008 & 0.003 \\
\hline ZEB2 & BRAF(A) & -0.216 & 0.01 & 0.854 \\
\hline SMARCA2 & SMARCA4(I) & -0.38 & 0.019 & 0.175 \\
\hline CTNNA1 & TP53(I),TP53(O) & 0.542 & 0.02 & 0.893 \\
\hline HRAS & HRAS(A) & -0.574 & 0.03 & 0.004 \\
\hline NFE2L2 & NFE2L2(A),KEAP1(O),KEAP1(I) & -0.317 & 0.051 & 0.000 \\
\hline SOX9 & BRAF(A) & -0.245 & 0.051 & 0.192 \\
\hline CDK6 & RB1(I) & 0.091 & 0.051 & 0.000 \\
\hline EFR3A & SMARCA4(I) & 0.065 & 0.055 & 0.032 \\
\hline TCF7L2 & APC(I),CTNNB1(A) & -0.312 & 0.072 & 0.000 \\
\hline MAP2K1 & BRAF(A) & -0.414 & 0.075 & 0.690 \\
\hline SEPHS2 & PIK3CA(A) & 0.094 & 0.125 & 0.714 \\
\hline E2F3 & RB1(I) & -0.295 & 0.167 & 0.001 \\
\hline MDM2 & BRAF(A),VHL(I),ARID5B(I) & -0.313 & 0.197 & 0.074 \\
\hline & & & & \\
\hline
\end{tabular}

Table S4: Associations between differential dependencies and sets of alterations identified by SuperDendrix. We rank results by FDR and the absolute value of SuperDendrix weight, $W(\bar{M})$. 
Table S5: Number of cell lines for each cancer type in the Avana and Score datasets.

Table S6: Associations between differential dependencies and sets of alterations/cancer types identified by SuperDendrix. Same format as Table S4

\begin{tabular}{|c|c|c|c|c|}
\hline Profile & Alterations and cancer types & $\mathbf{W}(\mathbf{M})$ & FDR & Validation P-value \\
\hline NRAS & NRAS(A) & -0.898 & 0 & 0 \\
\hline BRAF & BRAF(A) & -0.885 & 0 & 0 \\
\hline MYOD1 & Rhabdomyosarcoma & -0.872 & 0 & NA \\
\hline ISL1 & Neuroblastoma & -0.858 & 0 & 0 \\
\hline KRAS & KRAS(A) & -0.81 & 0 & 0 \\
\hline SOX10 & Skin & -0.781 & 0 & 0.103 \\
\hline MEF2C & Multiple myeloma,Leukemia & -0.774 & 0 & 0.003 \\
\hline MYB & Leukemia & -0.755 & 0 & 0.755 \\
\hline ZEB2 & Leukemia,Skin,Ewings sarcoma,Rhabdomyosarcoma & -0.75 & 0 & 0.182 \\
\hline MITF & Skin & -0.745 & 0 & 0.023 \\
\hline GFI1 & Leukemia & -0.736 & 0 & 0.036 \\
\hline IRF4 & Multiple myeloma,Lymphoma,Skin & -0.721 & 0 & 0.052 \\
\hline POU2AF1 & Multiple myeloma,MEF2B(A) & -0.713 & 0 & 0.004 \\
\hline HERPUD1 & Multiple myeloma & -0.699 & 0 & 0.006 \\
\hline IKZF1 & Leukemia,Lymphoma,Multiple myeloma & -0.697 & 0 & 0 \\
\hline HAND2 & Neuroblastoma & -0.689 & 0 & 0 \\
\hline IGF2BP1 & Ewings sarcoma,Neuroblastoma & -0.682 & 0 & 0.002 \\
\hline PRDM1 & Multiple myeloma & -0.681 & 0 & 0.251 \\
\hline MAPK1 & BRAF(A) & -0.662 & 0 & 0 \\
\hline FLI1 & Ewings sarcoma,Leukemia,Multiple myeloma & -0.661 & 0 & 0.249 \\
\hline PAX8 & Kidney,Endometrial,Ovarian & -0.661 & 0 & 0 \\
\hline ATP1B3 & Lymphoma,Multiple myeloma,Leukemia & -0.633 & 0 & 0 \\
\hline PEA15 & BRAF(A) & -0.622 & 0 & 0.011 \\
\hline SPDEF & Breast & -0.587 & 0 & 0.962 \\
\hline TP63 & Head and Neck,Bladder,Esophageal & -0.524 & 0 & 0 \\
\hline CCND3 & Leukemia,Lymphoma & -0.486 & 0 & 0.85 \\
\hline TCF7L2 & Colon,Gastric & -0.477 & 0 & 0 \\
\hline DUSP4 & Skin & -0.469 & 0 & 0.003 \\
\hline RAF1 & NRAS(A),KRAS(A) & -0.468 & 0 & 0 \\
\hline MYC & Neuroblastoma & 0.468 & 0 & 0 \\
\hline SPI1 & Leukemia & -0.461 & 0 & 0.393 \\
\hline PIK3CA & PIK3CA(A),Multiple myeloma & -0.453 & 0 & 0 \\
\hline PTPN11 & BRAF(A),NRAS(A),MYD88(O) & 0.439 & 0 & 0 \\
\hline GRB2 & BRAF(A),NRAS(A) & 0.419 & 0 & 0 \\
\hline NAMPT & Leukemia,Multiple myeloma,Breast,Lymphoma & -0.413 & 0 & 0.04 \\
\hline FERMT2 & Skin,Brain,Kidney,Ovarian,Sarcoma & -0.411 & 0 & 0 \\
\hline LDB1 & Leukemia,Neuroblastoma & -0.36 & 0 & 0 \\
\hline CDK6 & RB1(I),Breast,Endometrial,Ewings sarcoma,Ovarian & 0.351 & 0 & 0 \\
\hline SEPHS2 & Head and Neck,Colon,Brain,Esophageal,Gastric & 0.346 & 0 & 0 \\
\hline UMPS & Leukemia,Lymphoma,Multiple myeloma,Breast & -0.341 & 0 & 0.019 \\
\hline SHOC2 & NRAS(A),Brain & -0.339 & 0 & 0 \\
\hline GPX4 & Colon,Head and Neck,Esophageal,Skin,Gastric & 0.324 & 0 & 0 \\
\hline IRS2 & Ewings sarcoma,Neuroblastoma & -0.321 & 0 & 0.004 \\
\hline CFLAR & Skin,Brain,Neuroblastoma,KIT(O),Ewings sarcoma & 0.316 & 0 & 0.001 \\
\hline PSMB5 & Leukemia,Lymphoma,SMARCB1(I) & 0.296 & 0 & 0.124 \\
\hline CCND1 & RB1(I),Leukemia,Lymphoma,Rhabdomyosarcoma & 0.294 & 0 & 0 \\
\hline SNAP23 & Brain,Neuroblastoma,Skin,Ewings sarcoma & 0.292 & 0 & 0 \\
\hline EFR3A & SMARCA4(I),Neuroblastoma,Skin,Breast & 0.22 & 0 & 0 \\
\hline GFPT1 & Leukemia,VHL(I) & -0.19 & 0 & 0.013 \\
\hline
\end{tabular}




\begin{tabular}{|c|c|c|c|c|}
\hline DNM2 & Neuroblastoma & 0.187 & 0 & 0.015 \\
\hline PGM3 & Brain,Lung NSCLC & 0.175 & 0 & 0 \\
\hline TCF3 & Multiple myeloma,ID3(I),MEF2B(A) & -0.632 & 0.001 & 0.27 \\
\hline RELB & Multiple myeloma & -0.588 & 0.001 & 0.005 \\
\hline ARID1B & ARID1A(I) & -0.546 & 0.001 & 0.003 \\
\hline SLC5A3 & Leukemia & -0.449 & 0.001 & 0.089 \\
\hline STAG1 & STAG2(I) & -0.417 & 0.001 & $\overline{0.016}$ \\
\hline DOCK5 & KRAS(A) & -0.379 & 0.001 & 0 \\
\hline STX4 & TP53(I),TP53(O) & -0.369 & 0.001 & 0.177 \\
\hline CCND2 & Multiple myeloma & -0.325 & 0.001 & 0.003 \\
\hline TRPS1 & Breast & -0.51 & 0.002 & 0.003 \\
\hline GRHL2 & Head and Neck,Bladder,Breast,Pancreatic & -0.377 & 0.002 & 0.502 \\
\hline PTPN2 & Lymphoma & -0.588 & 0.002 & NA \\
\hline DCAF5 & Rhabdoid & -0.55 & 0.002 & NA \\
\hline BCL2 & Leukemia,Multiple myeloma,MEF2B(A),Neuroblastoma & -0.534 & 0.003 & 0 \\
\hline NFATC2 & Skin & -0.43 & 0.003 & 0.668 \\
\hline SMARCA2 & SMARCA4(I) & -0.38 & 0.003 & 0.189 \\
\hline ATP1A1 & Neuroblastoma & 0.147 & 0.003 & 0.002 \\
\hline ATP2C1 & Multiple myeloma & -0.349 & 0.004 & 0.005 \\
\hline CTNNA1 & TP53(I),TP53(O) & 0.542 & 0.005 & 0.982 \\
\hline TP53 & Rhabdoid,Kidney,Skin & 0.468 & 0.005 & 0.288 \\
\hline LMO2 & Leukemia & -0.323 & 0.005 & 0.198 \\
\hline PSTK & Colon,Esophageal,FLCN(I) & 0.177 & 0.005 & 0 \\
\hline YRDC & Brain & 0.079 & 0.005 & 0 \\
\hline SMAD7 & Multiple myeloma & -0.484 & 0.005 & 0.003 \\
\hline RPP25L & Brain,Lymphoma,Rhabdomyosarcoma & -0.248 & 0.006 & 0 \\
\hline TFAP2B & Neuroblastoma,Rhabdomyosarcoma & -0.551 & 0.006 & 0.006 \\
\hline RAB6A & SMARCA4(I),Skin & 0.19 & 0.006 & 0.005 \\
\hline MDM2 & Skin,Kidney,ARID5B(I) & -0.352 & 0.007 & 0.215 \\
\hline MET & Brain & -0.29 & 0.007 & 0.005 \\
\hline MARCH5 & Multiple myeloma,Leukemia & -0.288 & 0.007 & NA \\
\hline GET4 & Kidney & -0.159 & 0.007 & 0.122 \\
\hline BATF3 & Lymphoma,Multiple myeloma & -0.537 & 0.008 & 0.133 \\
\hline MYCN & Neuroblastoma,Rhabdomyosarcoma & -0.466 & 0.009 & 0.013 \\
\hline HRAS & HRAS(A) & -0.574 & 0.01 & 0.001 \\
\hline FOXA1 & Breast & -0.394 & 0.01 & 0.612 \\
\hline CHUK & Multiple myeloma,Lymphoma & -0.545 & 0.012 & 0.01 \\
\hline LIN28B & Neuroblastoma,Ewings sarcoma & -0.444 & 0.012 & 0.019 \\
\hline VCL & Brain & -0.237 & 0.012 & 0 \\
\hline SOX9 & Skin,Colon,Gastric,Pancreatic & -0.479 & 0.012 & 0.056 \\
\hline PAX3 & Rhabdomyosarcoma & -0.351 & 0.012 & NA \\
\hline IGF1R & Multiple myeloma,Ewings sarcoma,Rhabdomyosarcoma & -0.322 & 0.012 & 0.58 \\
\hline ARHGEF7 & KRAS(A),Head and Neck,Bladder,Liver & -0.415 & 0.016 & 0 \\
\hline NFE2L2 & NFE2L2(A),KEAP1(O),KEAP1(I) & -0.317 & 0.016 & 0.005 \\
\hline DHODH & Leukemia & -0.247 & 0.018 & 0.003 \\
\hline THAP1 & Leukemia,Lymphoma & 0.219 & 0.019 & 0.205 \\
\hline IKBKB & Multiple myeloma,Lymphoma & -0.607 & 0.02 & 0.006 \\
\hline IRS1 & Multiple myeloma,Rhabdomyosarcoma & -0.414 & 0.021 & 0.104 \\
\hline MAP2K1 & BRAF(A) & -0.414 & 0.021 & 0 \\
\hline TFAP2C & Breast & -0.341 & 0.022 & 0.103 \\
\hline ALG12 & Multiple myeloma,Lung & -0.49 & 0.031 & 0.015 \\
\hline CTNNB1 & Colon,Gastric,Liver,Pancreatic & -0.646 & 0.031 & 0 \\
\hline CAD & Leukemia,Multiple myeloma,Breast,Lymphoma & -0.304 & 0.031 & 0.009 \\
\hline EGFR & Head and Neck & -0.226 & 0.032 & 0 \\
\hline KCTD10 & TP53(I),TP53(O),Neuroblastoma,Rhabdoid & 0.25 & 0.032 & 0.44 \\
\hline GCLC & Leukemia,Lymphoma,Multiple myeloma,GATA3(I) & -0.634 & 0.037 & 0 \\
\hline
\end{tabular}




\begin{tabular}{|c|c|c|c|c|}
\hline AHCYL1 & Kidney,Liver & -0.172 & 0.037 & 0.251 \\
\hline E2F3 & RB1(I) & -0.295 & 0.042 & 0 \\
\hline JUN & Brain & -0.244 & 0.045 & 0 \\
\hline SEC23IP & Kidney & -0.25 & 0.049 & 0.926 \\
\hline HSPA13 & Head and Neck,Esophageal & -0.202 & 0.052 & 0 \\
\hline PTK2 & Skin,Brain & -0.186 & 0.055 & 0 \\
\hline PPAT & Leukemia & -0.151 & 0.065 & 0.008 \\
\hline CHEK2 & Kidney & 0.29 & 0.075 & 0.497 \\
\hline DMXL1 & Multiple myeloma,MEF2B(A) & -0.511 & 0.08 & 0.113 \\
\hline MVK & Brain & 0.081 & 0.08 & 0.008 \\
\hline FURIN & Multiple myeloma,Ewings sarcoma,Neuroblastoma,Leukemia,Pancreatic & -0.525 & 0.086 & 0.001 \\
\hline TFAP2A & Skin & -0.317 & 0.086 & 0.92 \\
\hline IPPK & Leukemia,Multiple myeloma,Lymphoma & -0.346 & 0.088 & 0.044 \\
\hline PPCDC & Leukemia,Lymphoma,Multiple myeloma & -0.328 & 0.088 & 0.4 \\
\hline SCAP & Neuroblastoma,ARID1B(I),Bone & 0.198 & 0.089 & 0 \\
\hline FPGS & Leukemia,Breast,Lymphoma & -0.276 & 0.102 & 0.08 \\
\hline PPARG & Bladder,Pancreatic & -0.442 & 0.106 & 0.399 \\
\hline WDR4 & Head and Neck & -0.176 & 0.106 & 0.025 \\
\hline FOSL1 & NF2(I),Head and Neck & -0.175 & 0.117 & 0 \\
\hline HNF1B & Kidney,Endometrial,Liver & -0.405 & 0.119 & 0.071 \\
\hline PARD6B & Kidney,Ovarian,Liver & -0.307 & 0.122 & 0 \\
\hline SEPSECS & Colon & 0.106 & 0.125 & 0.109 \\
\hline SMARCE1 & Rhabdoid & 0.122 & 0.136 & NA \\
\hline SMARCB1 & Rhabdoid,Sarcoma,Kidney & 0.302 & 0.141 & 0.172 \\
\hline EGLN1 & Skin,Ovarian & -0.337 & 0.15 & 0.321 \\
\hline RFK & Leukemia,Lymphoma,PBRM1(O),EPHA7(O) & -0.329 & 0.158 & 0.001 \\
\hline SLC4A7 & Lymphoma & -0.106 & 0.158 & NA \\
\hline PPP1R12A & Leukemia,Neuroblastoma & 0.226 & 0.161 & 0.002 \\
\hline ERBB2 & Head and Neck & -0.183 & 0.188 & 0.005 \\
\hline ZBTB38 & Multiple myeloma & -0.343 & 0.192 & 0.004 \\
\hline
\end{tabular}

Table S7: Enrichment for GO molecular functions in significant cancer-type dependencies.

Table S8: Enrichment for GO molecular functions in non-transcription factors with significant cancertype dependencies.

Table S9: List of 558 DepMap cell lines (2019Q1).

Table S10: Alteration dependencies from UNCOVER.

Table S11: Alteration or cancer-type dependencies from UNCOVER. 